\title{
SEMI-STABLE SUBCATEGORIES FOR EUCLIDEAN QUIVERS
}

\author{
COLIN INGALLS, CHARLES PAQUETTE, AND HUGH THOMAS \\ Dedicated to the memory of Dieter Happel
}

\begin{abstract}
In this paper, we study the semi-stable subcategories of the category of representations of a Euclidean quiver, and the possible intersections of these subcategories. Contrary to the Dynkin case, we find out that the intersection of semi-stable subcategories may not be semi-stable. However, only a finite number of exceptions occur, and we give a description of these subcategories. Moreover, one can attach a simplicial fan in $\mathbb{Q}^{n}$ to any acyclic quiver $Q$, and this simplicial fan allows one to completely determine the canonical presentation of any element in $\mathbb{Z}^{n}$. This fan has a nice description in the Dynkin and Euclidean cases: it is described using an arrangement of convex codimension-one subsets of $\mathbb{Q}^{n}$, each such subset being indexed by a real Schur root or a set of quasi-simple objects. This fan also characterizes when two different stability conditions give rise to the same semi-stable subcategory.
\end{abstract}

\section{INTRODUCTION}

Semi-stable subcategories. In Mumford's geometric invariant theory, in order to form the quotient of a variety by a group action, one first replaces the variety by its semi-stable points. This important idea was interpreted in the setting of quiver representations by King [22].

Let $Q$ be a (possibly disconnected) acyclic quiver, and $k$ an algebraically closed field. In this paper, all quivers are acyclic. We write $\operatorname{rep}(Q)$ for the category of finite-dimensional representations of $Q$ over $k$. For $\theta$, a $\mathbb{Z}$-linear functional on the Grothendieck group of $\operatorname{rep}(Q)$, referred to as a stability condition, King shows how to define the subcategory of $\theta$-semi-stable objects in $\operatorname{rep}(Q)$. It is immediate from King's definition, which we shall recall below, that for any $\theta$, the $\theta$-semistable subcategory of $\operatorname{rep}(Q)$ is abelian and extension-closed. It is therefore natural to ask which abelian and extension-closed subcategories can arise as semi-stable subcategories. (All subcategories considered in this paper are full subcategories. Also, when we refer to abelian subcategories, we mean exact abelian subcategories, that is to say, subcategories that are abelian with respect to the abelian structure of the ambient category.)

In $[19$, two of the authors of the present paper showed that when $Q$ is Dynkin, any abelian and extension-closed subcategory arises as the $\theta$-semi-stable subcategory of a suitable choice of $\theta$. In fact, somewhat more is known; see 19, Theorem 1.1]. For any acyclic quiver $Q$, the following conditions on an abelian, extensionclosed subcategory $\mathcal{A}$ of $\operatorname{rep}(Q)$ are equivalent; see Proposition 4.4 .

- $\mathcal{A}$ admits a projective generator.

- $\mathcal{A}$ is generated by the elements of an exceptional sequence.

1991 Mathematics Subject Classification. 16G20. 
- $\mathcal{A}$ is equivalent to the category of representations of some quiver $Q^{\prime}$.

We refer to subcategories satisfying these equivalent conditions as finitely generated. In 19, it was shown for any acyclic quiver $Q$, that any finitely generated, abelian and extension-closed subcategory of $\operatorname{rep}(Q)$ arises as the semi-stable subcategory for some stability condition. This resolves the Dynkin case because in that case, every abelian and extension-closed subcategory is finitely generated.

The next case one would hope to settle is the Euclidean case. In (ii) below, we refer to possibly disconnected Euclidean quivers. A possibly disconnected Euclidean quiver is defined to be a quiver with one Euclidean component and all other components (if any) Dynkin. A regular object is then a representation which is a direct sum of regular representations of the Euclidean component and/or representations of Dynkin components. We resolve the Euclidean case as follows:

Theorem 5.5. For $Q$ a Euclidean quiver, an abelian and extension-closed subcategory $\mathcal{B}$ of $\operatorname{rep}(Q)$ is the subcategory of $\theta$-semi-stable representations for some $\theta$ if and only if either:

(i) $\mathcal{B}$ is finitely generated, or

(ii) there exists some abelian, extension-closed, finitely generated subcategory $\mathcal{A}$ of $\operatorname{rep}(Q)$, equivalent to the representations of a Euclidean quiver (possibly disconnected), and $\mathcal{B}$ consists of all the regular objects of $\mathcal{A}$.

The semi-stable subcategories of the second type can also be described more explicitly as follows:

Proposition 9.12. The semi-stable subcategories in (ii) of Theorem 5.5 can also be described as those abelian, extension-closed subcategories of the regular part of $\operatorname{rep}(Q)$, which contain infinitely many indecomposable objects from each tube.

From [23, page 126], the category of coherent sheaves over a weighted projective line, in the tame domestic case, is derived-equivalent to $\operatorname{rep}(Q)$ for $Q$ Euclidean. Hence, we get the following, where two subcategories $\mathcal{B}_{1}, \mathcal{B}_{2}$ of a given hereditary abelian category $\mathcal{H}$ are orthogonal if for any objects $M_{1} \in \mathcal{B}_{1}, M_{2} \in \mathcal{B}_{2}$, $\operatorname{Hom}_{\mathcal{H}}\left(M_{1}, M_{2}\right)=0=\operatorname{Hom}_{\mathcal{H}}\left(M_{2}, M_{1}\right)$ and $\operatorname{Ext}_{\mathcal{H}}^{1}\left(M_{1}, M_{2}\right)=0=\operatorname{Ext}_{\mathcal{H}}^{1}\left(M_{2}, M_{1}\right)$.

Theorem 5.6. Let $\mathbb{X}$ be a weighted projective line of tame domestic type. Then an abelian and extension-closed subcategory $\mathcal{B}$ of $\operatorname{coh}(\mathbb{X})$ is the subcategory of $\theta$-semistable objects for some $\theta$ if and only if either:

(i) $\mathcal{B}$ is equivalent to the category of coherent sheaves over a weighted projective line,

(ii) $\mathcal{B}$ is equivalent to the torsion part of a category of coherent sheaves over a weighted projective line,

(iii) $\mathcal{B}$ is the additive hull of two abelian, extension-closed subcategories $\mathcal{B}_{1}, \mathcal{B}_{2}$ that are orthogonal to each other and where $\mathcal{B}_{1}$ is as in (i) or (ii) and $\mathcal{B}_{2}$ is of finite representation type.

Equivalence of stability conditions, canonical decomposition of dimension vectors. There is a great deal of interesting convex geometry associated to stability conditions. We can fix a dimension vector $\alpha$, and ask for what stability conditions $\sigma$ there will be objects of dimension vector $\alpha$ which are $\sigma$-semi-stable. This is shown to be a rational polyhedral cone [10, and these cones are studied further in 12. It is also of interest to consider the union of the cones that arise 
in this way; this has been done in [17, 8, where the emphasis was on the general theory and on the Dynkin case. We take a somewhat different approach: we draw similar pictures, but interpret them differently, and we focus on the Dynkin and Euclidean cases. They exhibit many interesting features that are absent from the wild setting. We consider all dimension vectors at once, focussing on the subcategory of semi-stable objects which each stability condition induces.

There is a natural equivalence relation on stability conditions, which we call ss-equivalence, where $\theta$ and $\theta^{\prime}$ are equivalent if they induce the same semi-stable subcategory. The set of stability conditions is naturally a free abelian group of finite rank, dual to the Grothendieck group, but in order to think about this equivalence, it is easier to work in the corresponding finite-dimensional vector space over $\mathbb{Q}$. It is also convenient to use the Euler form on the Grothendieck group to identify the Grothendieck group and its dual: we say that $d_{1}$ and $d_{2}$ are ss-equivalent if $\left\langle d_{1},-\right\rangle$ and $\left\langle d_{2},-\right\rangle$ are. (We recall the definition of the Euler form in Section 21)

Let $n$ denote the number of vertices of $Q$. There is a collection $\mathcal{J}$ of convex codimension-one subsets in $\mathbb{Q}^{n}$ such that $d_{1}$ and $d_{2}$ are ss-equivalent if and only if they lie in the same subsets of $\mathcal{J}$. Given an element $d \in \mathbb{Z}^{n}$, define

$$
\mathcal{J}_{d}=\{J \in \mathcal{J} \mid d \in J\} .
$$

Then we have:

Theorem 7.4, Let $Q$ be a Dynkin or Euclidean quiver and $d_{1}, d_{2} \in \mathbb{Z}^{n}$. Then $d_{1}$ and $d_{2}$ are ss-equivalent if and only if $\mathcal{J}_{d_{1}}=\mathcal{J}_{d_{2}}$.

Somewhat surprisingly, the geometry of $\mathcal{J}$, which controls ss-equivalence, can also be used to describe canonical decompositions, and more generally, canonical presentations in the sense of [17. Given a dimension vector for $Q$, it was shown by Kac 21] that the dimension vectors of the indecomposable summands of a generic representation of dimension vector $d$ are well-defined. The expression of $d$ as the sum of the dimension vectors of the indecomposable summands of a generic representation is called the canonical decomposition. In [17, the authors have extended this canonical decomposition to any element in $\mathbb{Z}^{n}$. If $d$ is a dimension vector, then the canonical presentation of $d$ coincides with the canonical decomposition of $d$. In general, one can write $d=d_{+}+d_{-}$, where $d_{+}$is a dimension vector and $-d_{-}$is the dimension vector of a projective representation whose top has a support disjoint from $d_{+}$. The canonical presentation of $d_{-}$is just the decomposition of $d_{-}$ as a (negative) linear combination of the dimension vectors of the indecomposable projective representations. Then the canonical presentation of $d$ is just the sum of the canonical decomposition of $d_{+}$and the canonical presentation of $d_{-}$. If the same summands appear in the canonical presentation of two vectors (possibly with different multiplicities), we say that the vectors are cp-equivalent. We show that cp-equivalence can also be characterized in terms of $\mathcal{J}$.

Let $\mathcal{L}$ be the set of all intersections of subsets of $\mathcal{J}$. We order $\mathcal{L}$ by inclusion. This is a lattice, which we call the intersection lattice associated to $\mathcal{J}$. For $L \in \mathcal{L}$, define the faces of $L$ to be the connected components of the set of points which are in $L$ but not in any smaller intersection. Define the faces of $\mathcal{L}$ to be the collection of all faces of all the elements of $\mathcal{L}$. Then we have the following theorem:

Theorem 8.3, Let $Q$ be a Dynkin or Euclidean quiver. Two vectors $d_{1}, d_{2}$ are cp-equivalent if and only if they lie in the same face of $\mathcal{L}$. 
Note that if two vectors $d_{1}, d_{2}$ lie in the same face of $\mathcal{L}$, then, in particular, $\mathcal{J}_{d_{1}}=\mathcal{J}_{d_{2}}$. It follows that if two dimension vectors are cp-equivalent, then they are ss-equivalent. This fact can also be established directly. The converse does not hold: if $\mathcal{J}_{d_{1}}=\mathcal{J}_{d_{2}}$, then the smallest element of $\mathcal{L}$ containing $d_{1}$ is also the smallest element of $\mathcal{L}$ containing $d_{2}$, but since elements of $\mathcal{L}$ are typically subdivided into more than one face, it does not follow that $d_{1}$ and $d_{2}$ lie in the same face, so they need not be cp-equivalent. Since cp-equivalence refines ss-equivalence, an ssequivalence class is a union of faces of $\mathcal{L}$.

Posets of subcategories. In Dynkin type, the semi-stable subcategories (or equivalently the abelian and extension-closed subcategories) form a lattice. It was shown in 19 that this poset is isomorphic to the lattice of noncrossing partitions associated to the Weyl group corresponding to $Q$. These lattices had already been studied by combinatorialists and group theorists and, especially relevant for our purposes, they play a central role in the construction of the dual Garside structure of Bessis [3] on the corresponding Artin group, which also leads to their use in constructing Eilenberg-Mac Lane spaces for the Artin groups [4, 5]. For these latter two uses, the lattice property of this partial order is essential.

For this reason, another motivation for our paper is to construct a potential replacement lattice in Euclidean type. The most obvious choice for a lattice associated to a general $Q$ would be to take all the abelian and extension-closed subcategories. However, this lattice is very big and somehow non-combinatorial; already in Euclidean type, it contains the Boolean lattice on the $\mathbb{P}^{1}(k)$-many tubes. On the other hand, we could consider finitely generated abelian and extension-closed subcategories. It was shown in [19] (Euclidean type) and [18] (general acyclic $Q$ ) that this yields the natural generalization of the noncrossing partitions of the associated Weyl group. However, for $Q$ non-Dynkin, this poset is typically not a lattice [14, which makes it unsuitable.

Therefore, in pursuit of a suitable lattice of subcategories associated to $Q$ it seems that we need to consider a class of subcategories which are not all finitely generated, but not so broad as to include the full plethora of abelian, extensionclosed subcategories. The lattice property can be guaranteed in a natural way if we can verify that our class of subcategories is closed under intersections. It turns out that the semi-stable subcategories do not form a lattice, but it is quite easy to describe the subcategories that arise as intersections of semi-stable subcategories; this class of subcategories then (automatically) forms a lattice. Specifically, we show:

Theorem 10.8 (simplified form). Let $Q$ be a Euclidean quiver. There are finitely many subcategories of $\operatorname{rep}(Q)$ which arise as an intersection of semi-stable subcategories, and which are not themselves semi-stable subcategories for any stability condition. These subcategories are contained entirely in the regular representations of $Q$. Any such subcategory can be written as the intersection of at most two semistable subcategories.

In fact, we give an explicit description of these subcategories, which we defer to the main body of the paper.

We hope to investigate the applicability of the lattice of these subcategories to the problem of constructing a dual Garside structure for Euclidean type Artin groups in subsequent work. 


\section{Some Representation theOry}

Let $Q=\left(Q_{0}, Q_{1}\right)$ be an (acyclic) quiver with $n$ vertices and let $k$ be an algebraically closed field. For simplicity, we shall assume that $Q_{0}=\{1,2, \ldots, n\}$. Our main concern is to study the semi-stable subcategories of $\operatorname{rep}(Q)$ and their intersections, when $Q$ is a Euclidean quiver. Unless otherwise specified, this includes the assumption that $Q$ is connected. When $Q$ is a Euclidean quiver, $\operatorname{rep}(Q)$ is of tame representation type and the representation theory of $Q$ is well understood. For the basic results concerning the structure of $\operatorname{rep}(Q)$, the reader is referred to [1, 27. We shall use many representation-theoretic results for $\operatorname{rep}(Q)$ and in particular, the structure of its Auslander-Reiten quiver.

Let $\langle$,$\rangle stand for the bilinear form defined on \mathbb{Z}^{n}$ as follows. If $d=\left(d_{1}, \ldots, d_{n}\right) \in$ $\mathbb{Z}^{n}$ and $e=\left(e_{1}, \ldots, e_{n}\right) \in \mathbb{Z}^{n}$, then

$$
\langle d, e\rangle=\sum_{i=1}^{n} d_{i} e_{i}-\sum_{\alpha: i \rightarrow j \in Q_{1}} d_{i} e_{j} .
$$

This is known as the Euler form associated to $Q$. This is a (non-symmetric) bilinear form defined on the Grothendieck group $K_{0}(\operatorname{rep}(Q))=\mathbb{Z}^{n}$ of $\operatorname{rep}(Q)$. For a representation $M$ in $\operatorname{rep}(Q)$, we denote by $d_{M}$ its dimension vector (which we identify with its class in the Grothendieck group). A crucial property of the above bilinear form is the following; see for example [1, Prop. III 3.13].

Proposition 2.1. Let $M, N \in \operatorname{rep}(Q)$. Then

$$
\left\langle d_{M}, d_{N}\right\rangle=\operatorname{dim}_{k} \operatorname{Hom}(M, N)-\operatorname{dim}_{k} \operatorname{Ext}^{1}(M, N) .
$$

This result justifies the terminology homological form that is sometimes used for the Euler form. We shall call a nonzero element $d=\left(d_{1}, \ldots, d_{n}\right)$ in $\mathbb{N}^{n}$ a dimension vector, as it is the dimension vector of some representation in $\operatorname{rep}(Q)$. It is sincere if $d_{i}>0$ for $i=1, \ldots, n$; and called a root if there exists an indecomposable representation $M$ with $d_{M}=d$. The previous statement implies that $\langle d, d\rangle \leq 1$; see [21]. If $d$ is a root with $\langle d, d\rangle=1$, then $d$ is called a real root, and otherwise, an imaginary root. If $d$ is a real root, then there is a unique, up to isomorphism, indecomposable representation having $d$ as a dimension vector; see 21. In this case, we write $M(d)$ for a fixed indecomposable representation with this dimension vector.

A root is called a Schur root if there exists an indecomposable representation $M$ with $d=d_{M}$ and such that $M$ has a trivial endomorphism ring. When $d$ is real, the latter condition is equivalent to $\operatorname{Ext}^{1}(M, M)=0$. An indecomposable representation having a trivial endomorphism ring is called a Schur representation.

We refer to an infinite component of the Auslander-Reiten quiver of $\operatorname{rep}(Q)$ which contains projective representations as a preprojective component (it is unique when $Q$ is connected and non-Dynkin); a preinjective component is defined similarly.

A real Schur root $d$ is called preprojective (resp. preinjective) if $M(d)$ lies in a preprojective (resp. preinjective) component of the Auslander-Reiten quiver of $\operatorname{rep}(Q)$. Otherwise, it is called regular.

Suppose now that $Q$ is a Euclidean quiver. Recall that a stable tube is a translation quiver obtained by taking the quotient of the translation quiver $\mathbb{Z} \mathbb{A}_{\infty}$ by some power of the translation. It is called a homogeneous tube when that power is one. A stable tube $T$ is standard if the full subcategory generated by the indecomposable 
representations in $T$ is equivalent to the path algebra $k T$ modulo the mesh relations. A representation in a stable tube which has only one arrow to and from it is called quasi-simple. For the convenience of the reader, the following proposition collects some results concerning the structure of the Auslander-Reiten quiver of $\operatorname{rep}(Q)$ and the morphisms between its connected components. All the statements can be found in [1] or [27].

Proposition 2.2. Let $Q$ be a Euclidean quiver. The Auslander-Reiten quiver of $\operatorname{rep}(Q)$ contains a preprojective component $\mathcal{P}$ of shape $\mathbb{N} Q^{\mathrm{op}}$, a preinjective component $\mathcal{I}$ of shape $\mathbb{N}^{-} Q^{\mathrm{op}}$, a $\mathbb{P}^{1}(k)$-family $\left\{T_{\lambda}\right\}$ of standard stable tubes of which only finitely many are non-homogeneous. Moreover, for $\lambda_{1}, \lambda_{2} \in \mathbb{P}^{1}(k)$, we have

(1) $\operatorname{Hom}(\mathcal{I}, \mathcal{P})=\operatorname{Hom}\left(\mathcal{I}, T_{\lambda_{1}}\right)=\operatorname{Hom}\left(T_{\lambda_{1}}, \mathcal{P}\right)=0$,

(2) $\operatorname{Hom}\left(T_{\lambda_{1}}, T_{\lambda_{2}}\right)=0$ if $\lambda_{1} \neq \lambda_{2}$.

Recall that in the Euclidean case, the quadratic form $q(x):=\langle x, x\rangle$ is positivesemidefinite, and its radical is of rank one and is generated by a dimension vector $\delta$. In particular, an imaginary root $d$ has the property that $\langle d, d\rangle=0$, and hence is also called isotropic. We shall call $\delta$ the null root of $Q$. Any isotropic (hence imaginary) root is a positive multiple of $\delta$. Observe moreover that $\delta$ is a Schur root and any other positive multiple of $\delta$ is also a root, but not a Schur root.

In the Euclidean case, the regular roots are the dimension vectors of the indecomposable representations which lie in a stable tube. Finally, observe that if $d$ is a root, then $\langle\delta, d\rangle$ is zero (resp. negative, positive) if and only if $d$ is regular (resp. preprojective, preinjective).

\section{Canonical decompositions, Semi-Invariants And SEmi-Stable SUBCATEGORIES}

In this section, $Q$ is any acyclic quiver, unless otherwise indicated. For a dimension vector $d=\left(d_{1}, \ldots, d_{n}\right)$, define $\operatorname{rep}(Q, d)$ to be the product of vector spaces specifying matrix entries for each of the arrows of $Q$. An element in $\operatorname{rep}(Q, d)$ is canonically identified with the corresponding representation in $\operatorname{rep}(Q)$ of dimension vector $d$. The reductive algebraic group $\mathrm{GL}_{d}(k):=\prod_{i=1}^{n} \mathrm{GL}_{d_{i}}(k)$ acts on $\operatorname{rep}(Q, d)$ by simultaneous change of basis and has a natural subgroup $\mathrm{SL}_{d}(k):=\prod_{i=1}^{n} \mathrm{SL}_{d_{i}}(k)$ which is a semi-simple subgroup. A semi-invariant for $\operatorname{rep}(Q, d)$ is just a polynomial function in $k[\operatorname{rep}(Q, d)]$ which is invariant under the action of $\mathrm{SL}_{d}(k)$. The set of semi-invariants for $\operatorname{rep}(Q, d)$ will be denoted $\operatorname{SI}(Q, d)$.

Now any linear map in $\operatorname{Hom}\left(\mathbb{Z}^{n}, \mathbb{Z}\right)$ is called a weight or a stability condition for $Q$. For $w \in \operatorname{Hom}\left(\mathbb{Z}^{n}, \mathbb{Z}\right)$ and an element $g=\left(g_{1}, \ldots, g_{n}\right)$ in $\mathrm{GL}_{d}(k)$, we set

$$
w(g)=w\left(g_{1}, \ldots, g_{n}\right)=\prod_{i=1}^{n} \operatorname{det}\left(g_{i}\right)^{w\left(e_{i}\right)},
$$

where for $1 \leq i \leq n$, the vector $e_{i}$ is the dimension vector of the simple representation at vertex $i$. In this way, when $d$ is sincere, the set of all weights for $Q$ corresponds to the set of all multiplicative characters for $\mathrm{GL}_{d}(k)$. Following King [22], an element $f \in k[\operatorname{rep}(Q, d)]$ is called a semi-invariant of weight $w$ if $g(f)=w(g) f$ for all $g=\left(g_{1}, \ldots, g_{n}\right)$ in $\mathrm{Gl}_{d}(k)$. We write $\operatorname{SI}(Q, d)_{w}$ for the set of semi-invariants of weight $w$ of $\operatorname{rep}(Q, d)$. 
When $d$ is sincere, the algebra $\operatorname{SI}(Q, d)$ is graded by the set of weights (which is the $\mathbb{Z}$-dual $K_{0}(Q)^{*}$ of $\left.K_{0}(Q)\right)$,

$$
\mathrm{SI}(Q, d)=\bigoplus_{w \text { weight }} \operatorname{SI}(Q, d)_{w} .
$$

Contrary to the way it is stated in 12, observe that when $d$ is not sincere, the direct sum is graded instead by $K_{0}\left(Q_{d}\right)^{*}$, where $Q_{d}$ is the full subquiver of $Q$ generated by the vertices in the support of $d$. Equivalently, it is more convenient to use $K_{0}(Q)^{*} / \sim_{d}$ as the grading set, where for $w, w^{\prime} \in K_{0}(Q)^{*}$, we have $w \sim_{d} w^{\prime}$ if $w, w^{\prime}$ agree on the elements supported over $Q_{d}$. In other words, if $f$ is a semi-invariant of weight $w$ in $\operatorname{SI}(Q, d)$ and $w \sim_{d} w^{\prime}$, then $f$ is also a semi-invariant of weight $w^{\prime}$, even if $w^{\prime} \neq w$. In the sequel, for a representation $M$, we sometimes write $\sim_{M}$ for $\sim_{d_{M}}$. We refer the reader to [12, 24, for fundamental results on semi-invariants of quivers. Let us just recall the results we need. Given two representations $M, N$, we have an exact sequence

$$
0 \rightarrow \operatorname{Hom}(M, N) \rightarrow \operatorname{Hom}\left(P_{0}, N\right) \stackrel{f_{N}^{M}}{\longrightarrow} \operatorname{Hom}\left(P_{1}, N\right) \rightarrow \operatorname{Ext}^{1}(M, N) \rightarrow 0
$$

where $0 \rightarrow P_{1} \rightarrow P_{0} \rightarrow M \rightarrow 0$ is the canonical projective resolution of $M$. We see that the $k$-linear map $f_{N}^{M}$ corresponds to a square matrix if and only if $\left\langle d_{M}, d_{N}\right\rangle=0$. In this case, we define $C^{M}(N)$ to be the determinant of $f_{N}^{M}$. Then $C^{M}(-)$, which is well defined up to a nonzero scalar, is a semi-invariant of weight $\left\langle d_{M},-\right\rangle$ for $\operatorname{rep}\left(Q, d_{N}\right)$. We see that $C^{M}(N) \neq 0$ if and only if $\operatorname{Hom}(M, N)=0=\operatorname{Ext}^{1}(M, N)$. The following result was proven in [10] for the general case and in [26] for the characteristic zero case.

Proposition 3.1. Let $w$ be a weight and $d$ a dimension vector. Then the vector space $\operatorname{SI}(Q, d)_{w}$ is generated over $k$ by the semi-invariants $C^{M}(-)$ where $\left\langle d_{M}, d\right\rangle=$ 0 and $w \sim_{d}\left\langle d_{M},-\right\rangle$.

Now, let us introduce the main object of study of this paper. Let $w$ be a weight. A representation $M$ in $\operatorname{rep}(Q)$ is said to be semi-stable with respect to $w$ or $w$-semistable if there exist $m>0$ and $f \in \operatorname{SI}\left(Q, d_{M}\right)_{m w}$ such that $f(M) \neq 0$. Given an element $d \in \mathbb{Z}^{n}$, write $\operatorname{rep}(Q)_{d}$ for the full subcategory of $\operatorname{rep}(Q)$ of the semi-stable representations with respect to the weight $\langle d,-\rangle$. We warn the reader here not to confuse $\operatorname{rep}(Q)_{d}$ with $\operatorname{rep}(Q, d)$, when $d$ is a dimension vector. These subcategories will be referred to as the semi-stable subcategories of $\operatorname{rep}(Q)$. If $Q^{\prime}$ is a full subquiver of $Q$ and $w$ is a weight for $Q$, then we denote by $\left.w\right|_{Q^{\prime}}: K_{0}\left(Q^{\prime}\right) \rightarrow \mathbb{Z}$ the weight of $Q^{\prime}$ which is the restriction of $w$. Given $M \in \operatorname{rep}(Q)$, denote by $\operatorname{Su}(M)$ its support and by $Q_{d_{M}}$ the full subquiver of $Q$ generated by $\mathrm{Su}(M)$. The following observation will be handy in the sequel.

Corollary 3.2. Let $M \in \operatorname{rep}(Q)$ and $d \in \mathbb{Z}^{n}$. Then $M \in \operatorname{rep}(Q)_{d}$ if and only if there exist a positive integer $m$ and a representation $V$ supported over $Q_{d_{M}}$ with $\left\langle d_{V},-\right\rangle \sim_{M}\langle m d,-\rangle$ and with $C^{V}(M) \neq 0$.

Proof. For the sufficiency, assume there exist a positive integer $m$ and a representation $V$ supported over $Q_{d_{M}}$ with $\left\langle d_{V},-\right\rangle \sim_{M}\langle m d,-\rangle$ and with $C^{V}(M) \neq 0$. Since $\left\langle d_{V},-\right\rangle \sim_{M}\langle m d,-\rangle$, we see that $\langle m d,-\rangle$ is a weight for $C^{V}(-)$ in $\operatorname{SI}\left(Q, d_{M}\right)$. Since $C^{V}(M) \neq 0$, we see that $M$ is $\langle d,-\rangle$-semi-stable. Assume now that $M \in \operatorname{rep}(Q)_{d}$. Then $M$ is identified with a representation $M^{\prime}$ of $Q_{d_{M}}$ and clearly, $M^{\prime}$ is $d^{\prime}-$ semi-stable in $\operatorname{rep}\left(Q_{d_{M}}\right)$, where $d^{\prime}=\left.\langle d,-\rangle\right|_{Q_{d_{M}}}$. Then, there exist $m>0$ and 
$f \in \operatorname{SI}\left(Q_{d_{M}}, d_{M}\right)_{m d^{\prime}}$ such that $f\left(M^{\prime}\right) \neq 0$. By Proposition 3.1 there exists a representation $V^{\prime} \in \operatorname{rep}\left(Q_{d_{M}}\right)$ with $C^{V^{\prime}}\left(M^{\prime}\right) \neq 0$ and $\left.\left\langle d_{V^{\prime}},-\right\rangle\right|_{Q_{d_{M}}}=m d^{\prime}$. Now, $V^{\prime}$ is identified with a representation $V$ of $\operatorname{rep}(Q)$ and we see that $C^{V}(M) \neq 0$.

Now, consider $K_{0}(\operatorname{rep}(Q)) \otimes_{\mathbb{Z}} \mathbb{Q}$, which will be identified with $\mathbb{Q}^{n}$. Given $d \in \mathbb{Q}^{n}$, let $[d]$ be the ray containing $d$ and emerging from the origin. We will denote by $\left(\mathbb{Q}^{n}\right)_{+}$the the positive orthant of $\mathbb{Q}^{n}$. For $d \in \mathbb{Z}^{n}$, we define $H_{d}$ to be the set of all elements $f$ in $\mathbb{Q}^{n}$ with $\langle f, d\rangle=0$ (observe that $H_{d}$ is a union of rays since $\langle-, d\rangle$ is homogeneous of degree one). We denote by $H_{d}^{+}$the intersection of $H_{d}$ with $\left(\mathbb{Q}^{n}\right)_{+}$. When $Q$ is a Euclidean quiver, one particularly important such set is $H_{\delta}$ defined by the equation $\langle-, \delta\rangle=0$ (or equivalently, of equation $\langle\delta,-\rangle=0$ ). As observed above, the roots in $H_{\delta}^{+}$are precisely the regular roots.

Now, let us recall the concept of canonical decomposition of a dimension vector defined by Kac [20; see also [12, 21. Let $d$ be a dimension vector. Suppose that there exists a non-empty open set $\mathcal{U}$ in $\operatorname{rep}(Q, d)$ and dimension vectors $d(1), \ldots, d(r)$ satisfying the following property: for each $M \in \mathcal{U}$, there exists a decomposition

$$
M \cong M_{1} \oplus \cdots \oplus M_{r}
$$

where each $M_{i}$ is indecomposable of dimension vector $d(i)$. (Note that the isomorphism class of $M_{i}$ may vary depending on the choice of $M \in \mathcal{U}$ - all that is fixed is the dimension vector.) In this case, we write

$$
d=d(1) \oplus \cdots \oplus d(r)
$$

and call this expression the canonical decomposition of $d$. The canonical decomposition always exists and is unique; see [20]. For more details concerning the canonical decomposition, we refer the reader to [12. Let us recall two fundamental results due to Kac and Schofield. Given two dimension vectors $d_{1}, d_{2}$, we set

$$
\operatorname{ext}\left(d_{1}, d_{2}\right)=\min \left(\left\{\operatorname{dim}_{k} \operatorname{Ext}^{1}\left(M_{1}, M_{2}\right) \mid d_{M_{1}}=d_{1}, d_{M_{2}}=d_{2}\right\}\right) .
$$

One also defines hom $\left(d_{1}, d_{2}\right)$ in an analogous way. The following result is due to Kac [21.

Proposition 3.3 (Kac). A decomposition

$$
d=d(1)+\cdots+d(m)
$$

where the $d(i)$ are dimension vectors corresponds to the canonical decomposition for $d$ if and only if the $d(i)$ are all Schur roots and $\operatorname{ext}(d(i), d(j))=0$ for $i \neq j$.

Actually, using the previous notations, a stronger statement holds; see [21]. There exists a non-empty open set $\mathcal{U}^{\prime}$ in $\operatorname{rep}(Q, d)$ such that for any $M \in \mathcal{U}^{\prime}$,

$$
M \cong M_{1} \oplus M_{2} \oplus \cdots \oplus M_{r}
$$

where each $M_{i}$ is a Schur representation of dimension vector $d(i)$ and we have $\operatorname{Ext}^{1}\left(M_{i}, M_{j}\right)=0$ if $i \neq j$. A representation $M$ which decomposes as a direct sum of pairwise Ext-orthogonal Schur representations will be called a general representation of dimension vector $d$. Hence, given a general representation $M$ of dimension vector $d$, one can recover the canonical decomposition of $d$ by looking at the dimension vectors of the indecomposable summands of $M$.

A dimension vector $d$ whose canonical decomposition only involves real Schur roots is called prehomogeneous. In such a case, there is a unique, up to isomorphism, 
general representation of dimension vector $d$. A fixed such representation will be denoted by $M(d)$ in the sequel. Note that $\operatorname{Ext}^{1}(M(d), M(d))=0$. When $d$ is a real Schur root, this notation agrees with our previous notation.

The following result, due to Schofield [25, shows how the canonical decomposition behaves when we multiply a dimension vector by a positive integer. For a Schur root $d$ and a positive integer $r$, let us define the following notation:

$$
d^{r}:= \begin{cases}d \oplus \cdots \oplus d(r \text { copies }), & \text { if } d \text { is real or isotropic } \\ r d, & \text { otherwise. }\end{cases}
$$

Proposition 3.4 (Schofield). Let $d$ be a dimension vector with canonical decomposition $d=d(1) \oplus \cdots \oplus d(r)$. For $m$ a positive integer, md has canonical decomposition $m d=d(1)^{m} \oplus \cdots \oplus d(r)^{m}$.

Observe that when $Q$ is of wild type and $s$ is a Schur imaginary non-isotropic root, then all the positive multiples of $s$ are also Schur imaginary roots. When $Q$ is a Dynkin or a Euclidean quiver, two distinct Schur roots lie on different rays in $\mathbb{Q}^{n}$.

Given a ray $r \in \mathbb{Q}^{n}$ with a dimension vector $d$ in it, one can consider the distinct rays $r_{1}, \ldots, r_{s}$ corresponding to the indecomposable summands of the canonical decomposition of $d$, and this is well defined by the above result of Schofield. In fact, we have the following.

Lemma 3.5. Let $d_{1}, d_{2}$ be two Schur roots and suppose that $d_{1}^{\prime} \in\left[d_{1}\right], d_{2}^{\prime} \in\left[d_{2}\right]$ are Schur roots. Then $\operatorname{ext}\left(d_{1}, d_{2}\right)=0$ if and only if $\operatorname{ext}\left(d_{1}^{\prime}, d_{2}^{\prime}\right)=0$.

Proof. We need only to prove the necessity and further, we need only to consider the case where at least one of the $d_{i}$ is imaginary and non-isotropic, say $d_{1}$. So assume that $\operatorname{ext}\left(d_{1}, d_{2}\right)=0$. Consider $d=d_{1}+d_{2}=d_{1} \oplus d_{2}$. We have to show that $d_{1}^{\prime} \oplus d_{2}^{\prime}$ is the canonical decomposition of $f:=d_{1}^{\prime}+d_{2}^{\prime}$. Let the canonical decomposition of $f$ be

$$
f=f(1) \oplus \cdots \oplus f(t) .
$$

There are positive integers $p_{1}, p_{2}, r_{1}, r_{2}$ such that $p_{1} d_{1}=r_{1} d_{1}^{\prime}$ and $p_{2} d_{2}=r_{2} d_{2}^{\prime}$. Let $\ell$ be the least common multiple of the $p_{i}$ and the $r_{i}$. From Proposition 3.4, we know that the canonical decomposition of $\ell f$ is

$$
\ell f=f(1)^{\ell} \oplus \cdots \oplus f(t)^{\ell} .
$$

Observe now that

$$
\begin{aligned}
\ell f & =\ell d_{1}^{\prime}+\ell d_{2}^{\prime} \\
& =t_{1} d_{1}+t_{2} d_{2}
\end{aligned}
$$

for some positive integers $t_{1}, t_{2}$. Using the canonical decomposition of $d$, we see that there are indecomposable Schur representations $M_{1}, M_{2}$, that are pairwise Ext-orthogonal such that $d_{M_{j}}=d_{j}$. Consider first the case where $d_{2}=d_{2}^{\prime}$ is either real or isotropic. Using these Schur representations, we see that the representations $M_{1}^{t_{1}}, M_{2}$ are pairwise Ext-orthogonal. Hence, using the definition of ext, we see that the Schur roots

$$
\left\{t_{1} d_{1}=\ell d_{1}^{\prime}, d_{2}\right\}
$$

are ext-orthogonal. Hence, from Proposition 3.3, the canonical decomposition of $\ell f$ is also given by

$$
\left(\ell d_{1}^{\prime}\right) \oplus d_{2}^{t_{2}} .
$$


By uniqueness of the canonical decomposition, we have

$$
f(1)^{\ell} \oplus \cdots \oplus f(t)^{\ell}=\left(\ell d_{1}^{\prime}\right) \oplus d_{2}^{t_{2}}
$$

from which it follows that $\left(\ell d_{1}^{\prime}\right)=(\ell f(j))$ for a unique $j$ and $d_{2}=f(i)$ for some $i$. This implies that $d_{1}^{\prime}=f(j)$ and $d_{2}^{\prime}=f(i)$. In particular, we get $\operatorname{ext}\left(d_{1}^{\prime}, d_{2}^{\prime}\right)=0$. Assume now that $d_{2}$ is imaginary and non-isotropic. The representations $M_{1}^{t_{1}}, M_{2}^{t_{2}}$ are pairwise Ext-orthogonal. Hence, we see that the Schur roots

$$
\left\{t_{1} d_{1}=\ell d_{1}^{\prime}, t_{2} d_{2}=\ell d_{2}^{\prime}\right\}
$$

are ext-orthogonal. Using the same argument as above, we see that $\left(\ell d_{1}^{\prime}\right)=(\ell f(j))$ for a unique $j$ and $\left(\ell d_{2}^{\prime}\right)=(\ell f(i))$ for a unique $i$. This implies that $d_{1}^{\prime}=f(j)$ and $d_{2}^{\prime}=f(i)$. In particular, we also get $\operatorname{ext}\left(d_{1}^{\prime}, d_{2}^{\prime}\right)=0$.

Now, let $r_{1}, r_{2}$ be two rays in $\left(\mathbb{Q}^{n}\right)_{+}$, each containing a Schur root. Then the condition ext $\left(r_{1}, r_{2}\right)=0$ does not depend on the particular choice of a root in each ray, and hence is well defined. Thus, we can make $\left(\mathbb{Q}^{n}\right)+$ into a simplicial fan whose rays are the rays associated to the Schur roots. Two rays $r_{1}, r_{2}$ associated to Schur roots belong to the same simplicial cone if and only if $\operatorname{ext}\left(r_{1}, r_{2}\right)=\operatorname{ext}\left(r_{2}, r_{1}\right)=0$. It is then clear that if $d$ is a dimension vector belonging to the relative interior of a simplicial cone generated by the rays $r_{1}, \ldots, r_{s}$, then the rays associated to the Schur roots in the canonical decomposition of $d$ are precisely $r_{1}, \ldots, r_{s}$. We call this simplicial fan on $\left(\mathbb{Q}^{n}\right)+$ the $c d$-fan.

We would like to extend the structure of simplicial fan on $\left(\mathbb{Q}^{n}\right)+$ to a structure of simplicial fan on the whole $\mathbb{Q}^{n}$. A similar construction is done in [17, but with less generality since not all imaginary Schur roots are considered. Let us give the construction. We denote by $p_{i}$ the dimension vector of the indecomposable projective representation at vertex $i, i=1,2, \ldots, n$. We add the rays $\left[-p_{i}\right]$ for $1 \leq i \leq n$ to the cd-fan. Let us define a compatibility relation on the set $\{[d] \mid$ $d$ is a Schur root $\} \cup\left\{\left[-p_{i}\right] \mid i=1,2, \ldots, n\right\}$ of rays. If $d_{1}, d_{2}$ are Schur roots, then $\left[d_{1}\right],\left[d_{2}\right]$ are compatible if $\operatorname{ext}\left(\left[d_{1}\right],\left[d_{2}\right]\right)=0$. If $d_{1}$ is a Schur root, then $\left[d_{1}\right],\left[-p_{i}\right]$ are compatible if $d_{1}$ is not supported at $i$. Finally, $\left[-p_{i}\right],\left[-p_{j}\right]$ for $i \neq j$ are always compatible. In this way, we can make $\mathbb{Q}^{n}$ into a simplicial fan such that if $d \in \mathbb{Z}^{n}$, then $d$ lies in the relative interior of a simplicial cone generated by some rays $r_{1}, \ldots, r_{s}$. The following proposition is essential for the definition of canonical presentation.

Proposition $3.6\left([17)\right.$. Let $d \in \mathbb{Z}^{n}$. There exists a decomposition

$$
d=t_{1} d_{1}+t_{2} d_{2}+\cdots+t_{m} d_{m}+t_{m+1} d_{m+1}+\cdots+t_{s} d_{s}
$$

where the $t_{i}$ are positive integers, $d_{1}, \ldots, d_{m}$ are $S$ chur roots, and $d_{m+1}, \ldots, d_{s}$ are negative of dimension vectors of indecomposable projective representations. Moreover, the $d_{i}, 1 \leq i \leq s$, are compatible in the sense defined above and $t_{i}=1$ whenever $d_{i}$ is an imaginary non-isotropic Schur root.

The decomposition given in Proposition 3.6 is called the canonical presentation of $d$. The positive part $t_{1} d_{1}+t_{2} d_{2}+\cdots+t_{m} d_{m}$ is denoted $d_{+}$while the negative part $t_{m+1} d_{m+1}+\cdots+t_{s} d_{s}$ is denoted $d_{-}$. Observe that $d_{+}$is a dimension vector whose canonical decomposition is $d_{+}=t_{1} d_{1}+\cdots+t_{m} d_{m}$ (or $d_{+}=d_{1}^{t_{1}} \oplus \cdots \oplus d_{m}^{t_{m}}$ using Schofield's notation) and $d_{-}$is equal to $-d_{P}$, where $P$ is a projective representation whose top has support disjoint to $d_{+}$. 
Example 3.7. Let $Q$ be the quiver $2 \rightarrow 1$. The Schur roots are the dimension vectors $(0,1),(1,0)$ and $(1,1)$, while the negative of the dimension vectors of the indecomposable projective representations are $(-1,0),(-1,-1)$. The figure below illustrates the fan for $Q$ that is defined above. The simplicial cones generated by two rays are denoted $C_{1}, C_{2}, C_{3}, C_{4}$ and $C_{5}$. The vector $d=(0,-1)$, shown in the diagram, lies in the relative interior of $C_{5}$. Therefore, we see that the canonical presentation of $d$ is $d=(1,0)+(-1,-1)$, where both coefficients are understood to be ones.

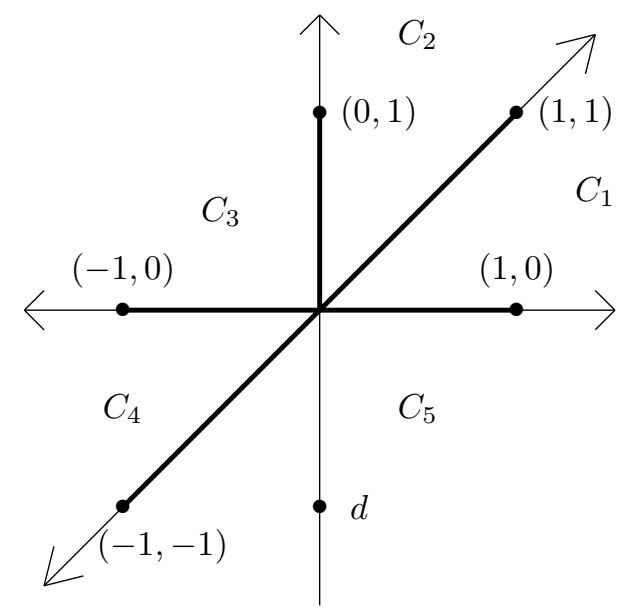

FiguRE 1. The above-defined fan for the quiver of type $\mathbb{A}_{2}$

The following lemma summarizes what we just discussed. It follows from our discussion and what appears in [17.

Lemma 3.8. For an acyclic quiver $Q$, the above-defined structure makes $\mathbb{Q}^{n}$ into a simplicial fan of dimension $n-1$. If $d \in \mathbb{Z}^{n}$ lies in the relative-interior of a simplicial cone generated by the rays $r_{1}, \ldots, r_{s}$, then let $d_{i} \in r_{i}$ be the smallest dimension vector.

(1) The vectors $d_{1}, \ldots, d_{s}$ are linearly independent.

(2) The canonical presentation of $d$ is $d=\sum_{i=1}^{s} t_{i} d_{i}$, where the $t_{i}$ are positive integers.

(3) The sum splits into two parts, where the summands in the first part add up to $d_{+}$, and the summands in the second part add up to $d_{-}$.

Note that in the above notation, if $d_{i}$ is imaginary and non-isotropic, then $t_{i} d_{i}$ means the Schur root $\left(t_{i} d_{i}\right)$ with a coefficient 1 in front. We will refer to this simplical fan on $\mathbb{Q}^{n}$ as the $c p$-fan. Observe that if we restrict this simplicial fan to the positive orthant, then we get the cd-fan.

\section{Exceptional obJects And EXCEPTIONAL SEQUences}

A representation $M$ in $\operatorname{rep}(Q)$ with $\operatorname{Ext}^{1}(M, M)=0$ is said to be rigid. If it is also indecomposable, it is called exceptional. It is well known (see for example [12]) that a representation $M$ is rigid if and only if its orbit in $\operatorname{rep}\left(Q, d_{M}\right)$ is open. A sequence 
of exceptional representations $\left(E_{1}, \ldots, E_{r}\right)$ is called an exceptional sequence if for all $i<j$, we have $\operatorname{Hom}\left(E_{i}, E_{j}\right)=0=\operatorname{Ext}^{1}\left(E_{i}, E_{j}\right)$. Note that some authors define an exceptional sequence using the "upper triangular" convention rather than the "lower triangular" convention that is used here.

The maximum length of an exceptional sequence in $\operatorname{rep}(Q)$ is the number of vertices in $Q$. Any exceptional sequence can be extended to one of maximal length; see $[9]$.

Given any full subcategory $\mathcal{V}$ of $\operatorname{rep}(Q)$, which may consist of a single object, we define $\mathcal{V}^{\perp}$ to be the full subcategory of $\operatorname{rep}(Q)$ generated by the representations $M$ for which $\operatorname{Hom}(V, M)=\operatorname{Ext}^{1}(V, M)=0$ for every $V \in \mathcal{V}$. We call $\mathcal{V}^{\perp}$ the right orthogonal category to $\mathcal{V}$. One also has the dual notion of left orthogonal category to $\mathcal{V}$, denoted ${ }^{\perp} \mathcal{V}$. We denote by $\mathcal{C}(\mathcal{V})$ the smallest abelian, extensionclosed subcategory of $\operatorname{rep}(Q)$ generated by the objects in $\mathcal{V}$. If $E$ is an exceptional sequence, and add $E$ its additive hull, we will abbreviate $\mathcal{C}(\operatorname{add} E)$ by $\mathcal{C}(E)$. We need the following well known fact. It was first proven by Geigle and Lenzing in [15], and later by Schofield in [24].

Proposition 4.1. Let $X$ be an exceptional representation in $\operatorname{rep}(Q)$, where $Q$ is an acyclic quiver. Then the category $X^{\perp}\left(\right.$ or $\left.{ }^{\perp} X\right)$ is equivalent to $\operatorname{rep}\left(Q^{\prime}\right)$ where $Q^{\prime}$ is an acyclic quiver having $\left|Q_{0}\right|-1$ vertices.

As an easy consequence, we have the following.

Proposition 4.2. Let $E=\left(X_{1}, \ldots, X_{r}\right)$ be an exceptional sequence in $\operatorname{rep}(Q)$ where $Q$ is an acyclic quiver. Then $\mathcal{C}(E)^{\perp}\left(\right.$ or $\left.{ }^{\perp} \mathcal{C}(E)\right)$ is equivalent to $\operatorname{rep}\left(Q^{\prime}\right)$ where $Q^{\prime}$ is an acyclic quiver having $\left|Q_{0}\right|-r$ vertices.

The following lemma is probably well known. We include a proof for completeness.

Lemma 4.3. Let $Q$ be a Euclidean quiver and $V, X$ be indecomposable representations with $X$ non-exceptional.

(1) If $V$ is preprojective, then $\operatorname{Hom}(V, X) \neq 0$.

(2) If $V$ is preinjective, then $\operatorname{Ext}^{1}(V, X) \neq 0$.

Proof. We only prove the second part. Suppose that $V$ is preinjective. There exists a non-negative integer $r$ with $\tau^{-r} V$ injective indecomposable. Hence, by the Auslander-Reiten formula,

$$
\operatorname{Ext}^{1}(V, X) \cong D \operatorname{Hom}(X, \tau V) \cong D \operatorname{Hom}\left(\tau^{-r-1} X, \tau^{-r} V\right) .
$$

Being non-exceptional, $X$ lies in a stable tube of the Auslander-Reiten quiver of $\operatorname{rep}(Q)$, and on or above the level in the tube consisting of representations whose dimension vector is the null root. It follows that the entire $\tau$-orbit of $X$ is sincere. Hence, $\tau^{-r-1} X$ is sincere. Since $\tau^{-r} V$ is injective indecomposable, we clearly have $\operatorname{Hom}\left(\tau^{-r-1} X, \tau^{-r} V\right) \neq 0$.

Proposition 4.4. Let $\mathcal{C}$ be an abelian, extension-closed subcategory of $\operatorname{rep}(Q)$. The following are equivalent.

(a) $\mathcal{C}$ is generated by an exceptional sequence,

(b) $\mathcal{C}$ is equivalent to $\operatorname{rep}\left(Q^{\prime}\right)$ for an acyclic quiver $Q^{\prime}$,

(c) $\mathcal{C}=V^{\perp}$ for some rigid representation $V$ of $\operatorname{rep}(Q)$. 
Proof. Suppose that $\mathcal{C}$ is generated by an exceptional sequence $E=\left(X_{r+1}, \ldots, X_{n}\right)$. Then, as was already remarked, $E$ can be completed to a full exceptional sequence

$$
E^{\prime}=\left(X_{1}, \ldots, X_{r}, X_{r+1}, \ldots, X_{n}\right) .
$$

Then $\mathcal{C}=\mathcal{C}\left(E^{\prime \prime}\right)^{\perp}$ where $E^{\prime \prime}=\left(X_{1}, \ldots, X_{r}\right)$ is an exceptional sequence. By Proposition 4.2, we get that $\cap_{i=1}^{r} X_{i}^{\perp}=\mathcal{C}\left(E^{\prime \prime}\right)^{\perp}$ is equivalent to $\operatorname{rep}\left(Q^{\prime}\right)$ for an acyclic quiver $Q^{\prime}$. This proves that $(a)$ implies $(b)$. The fact that $(b)$ implies $(a)$ follows from the fact that the module category of any triangular algebra is generated by an exceptional sequence, and an exceptional sequence for $\operatorname{rep}\left(Q^{\prime}\right)$ is also an exceptional sequence for $\operatorname{rep}(Q)$, since $\operatorname{rep}\left(Q^{\prime}\right)$ is an exact extension-closed subcategory of $\operatorname{rep}(Q)$.

Suppose now that $\mathcal{C}$ is generated by an exceptional sequence $E=\left(X_{r+1}, \ldots, X_{n}\right)$. As done in the first part of the proof, $E$ can be completed to a full exceptional sequence $E^{\prime}=\left(X_{1}, \ldots, X_{r}, X_{r+1}, \ldots, X_{n}\right)$. Then $E^{\prime \prime}=\left(X_{1}, \ldots, X_{r}\right)$ is an exceptional sequence and $\mathcal{C}\left(E^{\prime \prime}\right)$ is equivalent to $\operatorname{rep}\left(Q^{\prime}\right)$ for an acyclic quiver $Q^{\prime}$. Take $V$ a minimal projective generator of $\mathcal{C}\left(E^{\prime \prime}\right)$. Then $V$ is rigid and $\mathcal{C}(V)=\mathcal{C}\left(E^{\prime \prime}\right)$ with $V^{\perp}=\mathcal{C}\left(E^{\prime \prime}\right)^{\perp}=\mathcal{C}$. This proves that $(a)$ implies $(c)$. Finally, assume that $\mathcal{C}=V^{\perp}$ for a rigid representation $V$. Then $V$ is a partial tilting module and by Bongartz's lemma, it can be completed to a tilting module $V \oplus V^{\prime}$. Then $\operatorname{End}\left(V \oplus V^{\prime}\right)$ is a tilted algebra and hence has no oriented cycles in its quiver. In particular, the non-isomorphic indecomposable summands of $V$ form an exceptional sequence $E^{\prime \prime}=\left(Y_{1}, \ldots, Y_{s}\right)$ that can be completed to a full exceptional sequence $E^{\prime}=\left(Y_{1}, \ldots, Y_{s}, Y_{s+1}, \ldots, Y_{n}\right)$. Then $V^{\perp}=\mathcal{C}(E)$, where $E=\left(Y_{s+1}, \ldots, Y_{n}\right)$. This proves that $(c)$ implies $(a)$.

We can refine the previous proposition in the case that $Q$ is Euclidean. Recall our definition that a possibly disconnected Euclidean quiver is a quiver which has one Euclidean component and a finite number of Dynkin components. Similarly a possibly disconnected Dynkin quiver is a quiver whose connected components are all of Dynkin type.

Lemma 4.5. Let $Q$ be a Euclidean quiver and $V$ be a rigid representation. Then $V^{\perp}$ is equivalent to the representations of a (possibly disconnected) Euclidean quiver if $V$ is regular; otherwise, $V^{\perp}$ is equivalent to the representations of a (possibly disconnected) Dynkin quiver.

Proof. If $V$ is regular, then $V^{\perp}$ will include all the objects from the homogeneous tubes, and in particular, it will include representations whose dimension vector is the unique imaginary Schur root. Since we know $V^{\perp}$ is equivalent to the representations of some quiver, it must be equivalent to the representations of a (possibly disconnected) Euclidean quiver. (It clearly cannot be equivalent to the representations of a wild quiver, since it is contained in $\operatorname{rep}(Q)$.)

If $V$ is not regular, then let $Y$ be a non-regular indecomposable summand of $V$. Applying Lemma4.3. we see that $Y^{\perp}$ contains no non-exceptional indecomposables. Thus $V^{\perp} \subseteq Y^{\perp}$ must be of finite representation type.

\section{Description of Semi-Stable subcategories — Proof of Theorem 5.5}

In this section, we prove Theorem 5.5. We begin with $Q$ any acyclic quiver, and then specialize to the Euclidean case. Recall the following from [22]. 
Proposition 5.1 (King). Let $d \in \mathbb{Z}^{n}$. Then $M \in \operatorname{rep}(Q)$ is $\langle d,-\rangle$-semi-stable if $\left\langle d, d_{M}\right\rangle=0$ and whenever $M^{\prime}$ is a subrepresentation of $M$, then $\left\langle d, d_{M^{\prime}}\right\rangle \leq 0$.

The following proposition is well known, but we include a proof of our own.

Proposition 5.2. Suppose that $d$ is a prehomogeneous dimension vector and set $V=M(d)$. Then $\operatorname{rep}(Q)_{d}=\left\{M \in \operatorname{rep}(Q) \mid C^{V}(M) \neq 0\right\}=V^{\perp}$.

Proof. First, the fact that $\left\{M \in \operatorname{rep}(Q) \mid C^{V}(M) \neq 0\right\}=V^{\perp}$ follows from the exact sequence (11) and the definition of $C^{V}(M)$. Let $M \in \operatorname{rep}(Q)_{d}$. By Proposition 5.1 $\left\langle d, d_{M}\right\rangle=0$. In particular $\operatorname{dim}_{k} \operatorname{Hom}(V, M)=\operatorname{dim}_{k} \operatorname{Ext}^{1}(V, M)$. If both spaces are non-zero, then there exists a subrepresentation $M^{\prime}$ of $M$ with an epimorphism $V \rightarrow M^{\prime}$. This yields an epimorphism $\operatorname{Ext}^{1}(V, V) \rightarrow \operatorname{Ext}^{1}\left(V, M^{\prime}\right)$. Since $V$ is rigid, this gives $\operatorname{Ext}^{1}\left(V, M^{\prime}\right)=0$. But then, $\left\langle d, d_{M^{\prime}}\right\rangle=\operatorname{dim}_{k} \operatorname{Hom}\left(V, M^{\prime}\right)>0$, in contradiction to Proposition [5.1. Hence, $\operatorname{Hom}(V, M)=0=\operatorname{Ext}^{1}(V, M)$ which means that $M \in V^{\perp}$. Conversely, it is clear that if $M \in \operatorname{rep}(Q)$ with $C^{V}(M) \neq 0$, then $M \in \operatorname{rep}(Q)_{d}$.

Let us denote by $\mathcal{R}$ eg the full additive subcategory of $\operatorname{rep}(Q)$ generated by the indecomposable regular representations. When $Q$ is a Euclidean quiver, the canonical decomposition of a dimension vector only involves real Schur roots and possibly the null root $\delta$. We first need the following simple lemma about canonical decompositions.

Lemma 5.3. Let $Q$ be a Euclidean quiver. Let $d$ be a dimension vector which has the null root in its canonical decomposition. Then all the other summands of its canonical decomposition are regular.

Proof. Suppose that some real Schur root $\alpha$ appears in the canonical decomposition of $d$. Seeking a contradiction, suppose that $M(\alpha)$ is preinjective. We know by Proposition 3.3 that there exists an indecomposable with dimension vector $\delta$, say $V$, such that $\operatorname{Ext}^{1}(V, M(\alpha))=0=\operatorname{Ext}^{1}(M(\alpha), V)$. On the other hand, Lemma4.3 applied to $M(\alpha)$ and $V$ implies that $\operatorname{Ext}^{1}(M(\alpha), V) \neq 0$. A similar contradiction follows dually if $M(\alpha)$ is preprojective. Therefore $M(\alpha)$ is regular.

In the sequel, for a dimension vector $d$, we will denote by $\hat{d}$ the sum of the real Schur roots appearing in its canonical decomposition. The following result complements Proposition 5.2 in the Euclidean case.

Proposition 5.4. Let $Q$ be a Euclidean quiver. Suppose that $d$ is a dimension vector with canonical decomposition $d=\hat{d} \oplus \delta^{s}$ with $s \neq 0$. Set $V=M(\hat{d})$. Then

$$
\operatorname{rep}(Q)_{d}=\left\{M \in \operatorname{rep}(Q) \mid C^{V}(M) \neq 0\right\} \cap \mathcal{R} \text { eg }=V^{\perp} \cap \mathcal{R} \text { eg. }
$$

Proof. By Proposition [5.2, we only need to prove the first equality. Let $M(\delta)$ be a general representation of dimension vector $\delta$. Then $V \oplus M(\delta)^{s}$ is a general representation of dimension vector $d$. By Lemma 5.3. we see that $V$ is regular. Let $M \in \operatorname{rep}(Q)_{d}$ be indecomposable. Then $\left\langle d, d_{M}\right\rangle=0$. If $M$ is preinjective, then $\operatorname{Ext}^{1}(M(\delta) \oplus V, M)=0$, and hence $0=\left\langle d, d_{M}\right\rangle=\operatorname{dim}_{k} \operatorname{Hom}\left(V \oplus M(\delta)^{s}, M\right)$. But $\operatorname{Hom}(M(\delta), M) \neq 0$ by Lemma 4.3, so we get a contradiction. Similarly, $M$ cannot be preprojective. Therefore, $M$ is regular. We may assume that $\operatorname{Hom}(M(\delta), M)=$ $0=\operatorname{Ext}^{1}(M(\delta), M)$. This gives $\left\langle d, d_{M}\right\rangle=\operatorname{dim}_{k} \operatorname{Hom}(V, M)-\operatorname{dim}_{k} \operatorname{Ext}^{1}(V, M)=0$. Assume $\operatorname{Hom}(V, M) \neq 0$. There exists an epimorphism $V \rightarrow L$ for some subrepresentation $L$ of $M$. Since we have an epimorphism $\operatorname{Ext}^{1}(V, V) \rightarrow \operatorname{Ext}^{1}(V, L)$ and $V$ 
is rigid, we see that $\operatorname{Ext}^{1}(V, L)=0$. Since $\left\langle d, d_{L}\right\rangle \leq 0$, this gives $\operatorname{Hom}(V, L)=0$, a contradiction. Therefore, $\operatorname{Hom}(V, M)=0$ and hence, $M \in V^{\perp}$. This shows $\operatorname{rep}(Q)_{d} \subseteq V^{\perp} \cap \mathcal{R}$ eg. Conversely, assume that $M \in V^{\perp} \cap \mathcal{R e g}$. First, $M \in V^{\perp}$ is clearly equivalent to $C^{V}(M) \neq 0$. Since $M$ is regular, $M$ lies in a stable tube. Hence, there exists a quasi-simple representation $W$ lying in a homogeneous tube (and hence having dimension vector $\delta$ ) with $\operatorname{Hom}(W, M)=\operatorname{Ext}^{1}(W, M)=0$. We therefore have $C^{V \oplus W^{s}}(M) \neq 0$, which shows that $M \in \operatorname{rep}(Q)_{d}$.

We can now prove our first theorem from the introduction. Recall that for a possibly disconnected Euclidean quiver $Q$, the indecomposable regular representations are defined to be the regular representations of the Euclidean component together with the indecomposable representations of the Dynkin components.

Theorem 5.5. For $Q$ a Euclidean quiver, an abelian and extension-closed subcategory $\mathcal{B}$ of $\operatorname{rep}(Q)$ is the subcategory of $\theta$-semi-stable representations for some $\theta$ if and only if either:

(i) $\mathcal{B}$ is finitely generated, or

(ii) there exists some abelian, extension-closed, finitely generated subcategory $\mathcal{A}$ of $\operatorname{rep}(Q)$, equivalent to the representations of a Euclidean quiver (possibly disconnected), and $\mathcal{B}$ consists of all the regular objects of $\mathcal{A}$.

Proof. First, write $\theta=\langle d,-\rangle$. If $d$ is not a dimension vector, then it follows from Corollary 3.2 that $\operatorname{rep}(Q)_{d}$ is supported over a proper subquiver of $Q$, and hence it is an abelian extension-closed subcategory of a category of representations of a (possibly disconnected) Dynkin quiver and hence it satisfies (i). So assume that $d$ is a dimension vector. Suppose the canonical decomposition of $d$ does not include the null root. Then by Proposition 5.2 , we know that $\operatorname{rep}(Q)_{d}$ is of the form $V^{\perp}$ for some rigid representation $V$. By Proposition 4.4 this implies that it is finitely generated.

Suppose the canonical decomposition of $d$ does include the null root. By Proposition 5.4, we know that the semi-stable category corresponding to $d$ is of the form $V^{\perp} \cap \mathcal{R}$ eg. By Lemma [5.3, we can take $V$ to be regular and rigid. By Lemma 4.5. $V^{\perp}$ is equivalent to the representations of a (possibly disconnected) Euclidean quiver. The subcategory is therefore of type (ii).

Conversely, suppose that we have a finitely generated abelian and extensionclosed subcategory $\mathcal{B}$ of $\operatorname{rep}(Q)$. It is generated by an exceptional sequence, say $\left(X_{r+1}, \ldots, X_{n}\right)$ which can be extended to a full exceptional sequence $\left(X_{1}, \ldots, X_{n}\right)$. Let $\mathcal{F}$ be the extension-closed abelian subcategory generated by $\left(X_{1}, \ldots, X_{r}\right)$, and let $P$ be a projective generator of $\mathcal{F}$. Then $\mathcal{B}=P^{\perp}$. Since $P$ is partial tilting, it is the general representation of dimension $d_{P}$. Therefore the semi-stable subcategory associated to $d_{P}$ is $\mathcal{B}$ by Proposition 5.2 .

Finally, suppose that we have an extension-closed abelian subcategory $\mathcal{B}$ as in (ii), which consists of the regular objects in some finitely generated abelian and extension-closed subcategory $\mathcal{A}$, with $\mathcal{A}$ equivalent to the representations of some (possibly disconnected) Euclidean quiver. As in the previous case, we know that $\mathcal{A}$ can be written as $P^{\perp}$. Since $\mathcal{A}$ is of (possibly disconnected) Euclidean type, $P$ must be regular by Lemma 4.5. Hence, an indecomposable summand of $P$ is either in a stable tube or is supported by a Dynkin component of the quiver. By Proposition 3.3. we know that a general representation of dimension vector $\delta+d_{P}$ will be isomorphic to a direct sum of $P$ and an indecomposable representation of 
dimension vector $\delta$. Therefore the semi-stable subcategory associated to $\delta+d_{P}$ will be $\mathcal{B}$ by Proposition 5.4

Note that from the previous theorem, not all abelian and extension-closed subcategories of $\operatorname{rep}(Q)$ are semi-stable, and that a semi-stable subcategory may be disconnected. In [13], there is a description of all the connected abelian and extensionclosed subcategories of $\operatorname{rep}(Q)$.

The previous theorem can be interpreted for the category $\operatorname{coh}(\mathbb{X})$ of coherent sheaves over a weighted projective line $\mathbb{X}$ of positive Euler characteristic (that is, of domestic tame type). The reader is referred to [23] for an introduction to coherent sheaves over a weighted projective line. It is well known that when $\mathbb{X}$ is a weighted projective line of tame domestic type, then $\operatorname{coh}(\mathbb{X})$ is derived equivalent to $\operatorname{rep}(Q)$ for $Q$ a Euclidean quiver; see [23, page 126]. The abelian category $\operatorname{coh}(\mathbb{X})$ is also equivalent to the category of sheaves on a Fano orbifold with coarse moduli space $\mathbb{P}^{1}$, and to the category of sheaves of modules on a Fano hereditary order as can be seen, for example, in [6], 7]. Here Fano is equivalent to the fact that $\sum\left(1-1 / r_{i}\right)<2$ where the $r_{i}$ represent the widths of the non-homogeneous tubes, the orders of the non-trivial stabilizers of the orbifold, or the ramification indices of the hereditary order. Hence, we can immediately derive the following as a corollary of the previous theorem.

Theorem 5.6. Let $\mathbb{X}$ be a weighted projective line of tame domestic type. Then an abelian and extension-closed subcategory $\mathcal{B}$ of $\operatorname{coh}(\mathbb{X})$ is the subcategory of $\theta$-semistable objects for some $\theta$ if and only if either:

(i) $\mathcal{B}$ is equivalent to the category of coherent sheaves over a weighted projective line,

(ii) $\mathcal{B}$ is equivalent to the torsion part of a category of coherent sheaves over a weighted projective line,

(iii) $\mathcal{B}$ is the additive hull of two abelian, extension-closed subcategories $\mathcal{B}_{1}, \mathcal{B}_{2}$ that are orthogonal to each other and where $\mathcal{B}_{1}$ is as in (i) or (ii) and $\mathcal{B}_{2}$ is of finite representation type.

\section{Canonical DeComposition on the Regular hyperplane}

In this section, $Q$ is assumed to be a (connected) Euclidean quiver. Let us introduce some notation. Note that a representation is quasi-simple if it is a simple object in the full subcategory of $\operatorname{rep}(Q)$ consisting of the regular representations. For convenience, a regular real Schur root which corresponds to a quasi-simple representation will be called a quasi-simple root. Hence, the null root is not considered to be a quasi-simple root here. We define $H_{\delta}^{s s} \subseteq H_{\delta}^{+}$to be the convex cone in $\mathbb{Q}^{n}$ generated by the quasi-simple roots and $\delta$. In particular, $H_{\delta}^{s s} \subseteq\left(\mathbb{Q}^{n}\right)_{+}$. We call it the regular cone of $Q$, or of $\mathbb{Q}^{n}$. Our goal in this section is to describe the geometry of $H_{\delta}^{s s}$ and the structure of the canonical decomposition for $d \in H_{\delta}^{s s}$. The reader is referred to [2] for the basic notions on convex geometry that are used in this section.

We label the non-homogeneous tubes in the Auslander-Reiten quiver of $\operatorname{rep}(Q)$, by $1, \ldots, N$. For $1 \leq i \leq N$, let $r_{i}$ be the rank of the tube labeled $i$ and let $\beta_{i 1}, \ldots, \beta_{i r_{i}}$ be the quasi-simple roots in that tube. It is well known (see [27. 
Theorem XIII 2.1]) that

$$
\sum_{i=1}^{N}\left(r_{i}-1\right)=n-2
$$

Hence there are exactly $n-2+N$ quasi-simple roots. If $d_{1}, d_{2}$ are roots corresponding to the indecomposable representations $M_{1}, M_{2}$, respectively, and $M_{2}$ is the Auslander-Reiten translate of $M_{1}$, then we write $\tau\left(d_{1}\right)=d_{2}$ and say that $d_{2}$ is the Auslander-Reiten translate of $d_{1}$. If $C$ denotes the Coxeter transformation, this just means that $d_{2}=C\left(d_{1}\right)$.

Lemma 6.1. The space of linear dependencies among the vectors $\beta_{i j}$ is $(N-1)$ dimensional. The linear dependencies are spanned by those that arise from the fact that the sum of the quasi-simple roots of any non-homogeneous tube is equal to that of any other non-homogeneous tube.

Proof. It is well known that each tube contains representations whose dimension vector is the null root and that these representations have a filtration by quasisimples in which each quasi-simple in the tube appears exactly once. This implies that the sum of the quasi-simple roots in any tube equals the null root. This gives rise to $(N-1)$ linearly independent dependencies among the vectors $\beta_{i j}$. We need therefore only verify that there are no additional dependencies.

Let $\sum c_{i j} \beta_{i j}=0$ for some constants $c_{i j}$. Pick some tube with index $i_{0}$ and some quasi-simple with index $j_{0}$. There are two dimension vectors of quasi-simples which have non-zero pairing with $\beta_{i_{0} j_{0}}$, namely $\beta_{i_{0} j_{0}}$ and its inverse AR translate. Specifically,

$$
\left\langle\beta_{i_{0} j_{0}}, \beta_{i_{0} j_{0}}\right\rangle=1 \text { and }\left\langle\tau^{-1} \beta_{i_{0} j_{0}}, \beta_{i_{0} j_{0}}\right\rangle=-1 .
$$

Since $\sum c_{i j} \beta_{i j}=0$, we must have that $\left\langle\sum c_{i j} \beta_{i j}, \beta_{i_{0} j_{0}}\right\rangle=0$. It follows that the coefficients of $\beta_{i_{0} j_{0}}$ and $\tau^{-1} \beta_{i_{0} j_{0}}$ must be equal. By considering all possible choices of $j_{0}$ for the tube $i_{0}$, we see that all the coefficients corresponding to quasi-simples from tube $i_{0}$ must be equal. The same argument applies to the other tubes, and thus the linear dependence which we started with lies in the span of those we found initially.

To describe the facets of $H_{\delta}^{s s}$, we introduce some notation. If $\left|Q_{0}\right|>2$, write $R$ for the set of $N$-tuples $\left(a_{1}, \ldots, a_{N}\right)$ with $1 \leq a_{i} \leq r_{i}$. For $\left(a_{1}, \ldots, a_{N}\right) \in R$, write $F_{\left(a_{1}, \ldots, a_{N}\right)}$ for the convex cone in $\left(\mathbb{Q}^{n}\right)_{+}$generated by the quasi-simple roots except for $\beta_{i a_{i}}$ for $1 \leq i \leq N$.

Proposition 6.2. Suppose that $\left|Q_{0}\right|>2$. The boundary facets of $H_{\delta}^{s s}$ are exactly the $F_{I}$ for $I \in R$.

Proof. For $1 \leq i \leq N$, write $P_{i}$ for the cone generated by the quasi-simples from the $i$-th tube. From Lemma 6.1, we see that the dimension vectors of the quasisimple representations in a given tube are linearly independent. Therefore, $P_{i}$ is a simplicial cone containing the ray generated by $\delta$.

Consider the $(n-1)$-dimensional vector space $\mathbb{Q}^{n} /\langle\delta\rangle$, and let $\bar{P}_{i}$ be the subspace of this quotient space generated by $P_{i}$. By Lemma 6.1, the $\bar{P}_{i}$ are complementary. It follows that codimension one facets of $H_{\delta}^{s s}$ are each formed by taking the convex hull of one facet from each $P_{i}$. The description of the facets given in the statement of the proposition then follows. 
If $\Sigma_{1}$ and $\Sigma_{2}$ are two simplicial complexes on disjoint vertex sets $V_{1}, V_{2}$, then the simplicial join of $\Sigma_{1}$ and $\Sigma_{2}$ is defined by saying that a set $F \subseteq V_{1} \cup V_{2}$ is a face if and only if $F \cap V_{1}$ is a face of $\Sigma_{1}$ and $F \cap V_{2}$ is a face of $\Sigma_{2}$. In the sequel, we will use the simplicial join of at most three simplices. Some low dimensional examples include a square, an octahedron and a triangular bipyramid.

Corollary 6.3. Suppose that $\left|Q_{0}\right|>2$. The combinatorial structure of the boundary of $H_{\delta}^{s s}$ can be described as a cone over the simplicial join of the boundary of $N$ simplices, one for each non-homogeneous tube, where the $i$-th simplex has as vertices the quasi-simples from the $i$-th tube.

Example 6.4. Let $Q$ be the quiver below:

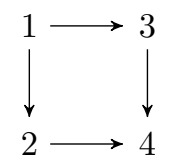

The following is an affine slice of $\left(\mathbb{Q}^{n}\right)_{+}$, with $H_{\delta}^{s s}$ indicated:

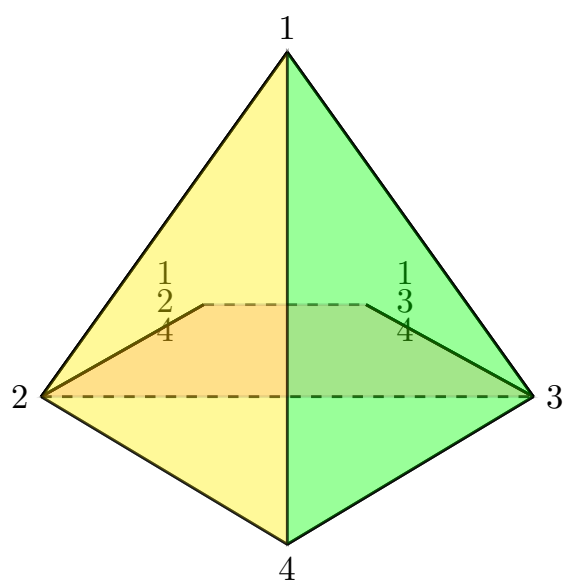

Observe that $H_{\delta}^{s s}$ is not equal to the intersection of the affine span of $H_{\delta}$ with $\left(\mathbb{Q}^{n}\right)_{+}$in this case (contrary to what one might have expected from smaller examples). For instance, the dimension vector $d_{S_{1}}+d_{S_{4}}$ lies in $H_{\delta}$ but not in $H_{\delta}^{s s}$.

Corollary 6.5. All the regular real Schur roots lie on the boundary of $H_{\delta}^{s s}$.

Proof. Using the fact that the stable tubes are standard (see Proposition 2.2) and that for $X$ regular, $\operatorname{Ext}^{1}(X, X) \cong D \operatorname{Hom}(X, \tau X)$, we get that the regular real Schur roots correspond to representations which admit a filtration by quasi-simples from some tube which does not include all the quasi-simples from that tube. It therefore follows that each real Schur root lie on the boundary of some $P_{i}$ (defined in the proof of Proposition 6.2), and thus on the boundary of $H_{\delta}^{s s}$.

For $Q$ the Kronecker quiver, that is, when $\left|Q_{0}\right|=2$, we define $R$ to be the set with one element $\emptyset$ and we set $F_{\emptyset}=\emptyset$. 
For each facet $F_{I}$ of $H_{\delta}^{s s}$, denote by $C_{I}$ the simplicial cone in $\left(\mathbb{Q}^{n}\right)_{+}$generated by $F_{I}$ and $\delta$. Note that in the Kronecker case, there is a unique cone $C_{\emptyset}$, and it is equal to the ray generated by $\delta$.

Proposition 6.6. Let $d$ be a dimension vector lying in $H_{\delta}^{s s}$.

(1) If d lies on a facet $F_{I}$ of $H_{\delta}^{s s}$, then the canonical decomposition of d only involves regular real Schur roots in $F_{I}$.

(2) If $d$ lies in the relative interior of $H_{\delta}^{s s}$, say in $C_{I}$, then the canonical decomposition of $d$ involves the null root and possibly some regular real Schur roots in $F_{I}$.

Proof. From what we just proved, we have that $\delta$ lies in the relative interior of $H_{\delta}^{s s}$. Moreover, all the quasi-simple roots lie on the boundary of $H_{\delta}^{s s}$.

Suppose first that $d$ is a dimension vector lying on the facet $F_{I}$ of $H_{\delta}^{s s}$. The additive extension-closed abelian subcategory of $\operatorname{rep}(Q)$ generated by the quasisimple representations corresponding to the roots in $F_{I}$ is equivalent to the category of representations of a quiver which is a union of quivers of type $\mathbb{A}$. This observation, together with Proposition 3.3, gives that the canonical decomposition of $d$ only involves real Schur roots lying on $F_{I}$.

Suppose now that $d$ lies in the relative interior of $H_{\delta}^{s s}$, say in $C_{I}$. We can decompose $d$ as $d=d_{1}+r \delta$ where $d_{1} \in F_{I}$ and $r \in \mathbb{Z}$. Let $d_{1}=d(1) \oplus \cdots \oplus d(m)$ be the canonical decomposition of $d_{1}$ which, by the above argument, only involves real Schur roots in $F_{I}$. By Proposition 3.3, it is clear that

$$
d=d(1) \oplus \cdots \oplus d(m) \oplus \delta^{r}
$$

is the canonical decomposition of $d$ since $\operatorname{ext}(d(i), \delta)=\operatorname{ext}(\delta, d(i))=0$ for all $i$.

\section{Ss-equivalence and PRoOf of Theorem 7.4}

In this section, we suppose that $Q$ is a Dynkin or Euclidean quiver with $n$ vertices, unless otherwise indicated. We prove Theorem 7.4 which describes when two dimension vectors determine the same semi-stable subcategories.

Recall that when $Q$ is Euclidean, the quadratic form $q(x)=\langle x, x\rangle$ is positive semi-definite and its radical is of rank one, generated by the null root $\delta$. Recall also that the convex set $H_{\delta}^{s s}$ is defined as the cone generated by the quasi-simple roots, if $Q$ is of Euclidean type and contains more than 2 vertices, and $H_{\delta}^{s s}=\{[\delta]\}$, if $Q$ is the Kronecker quiver.

Let $X$ be an exceptional representation in $\operatorname{rep}(Q)$ and from Proposition 4.1 let $Q_{X}$ be the quiver with $n-1$ vertices for which ${ }^{\perp} X \cong \operatorname{rep}\left(Q_{X}\right)$. Note that $Q_{X}$ is a possibly disconnected Euclidean or Dynkin quiver. Let

$$
F_{d_{X}}:{ }^{\perp} X \rightarrow \operatorname{rep}\left(Q_{X}\right)
$$

be an exact functor which is an equivalence. Denote by $G_{d_{X}}$ a quasi-inverse functor. Let $S_{1}^{X}, S_{2}^{X}, \ldots, S_{n-1}^{X}$ be the non-isomorphic simple objects in ${ }^{\perp} X$, called the relative simples in ${ }^{\perp} X$. It is clear that $K_{0}\left({ }^{\perp} X\right)$, which is isomorphic to $K_{0}\left(\operatorname{rep}\left(Q_{X}\right)\right)$, is the subgroup of $K_{0}(\operatorname{rep}(Q))$ generated by the classes $\left[S_{1}^{X}\right], \ldots,\left[S_{n-1}^{X}\right]$ in $K_{0}(\operatorname{rep}(Q))$. Put

$$
\varphi_{X}: K_{0}\left({ }^{\perp} X\right) \rightarrow K_{0}\left(\operatorname{rep}\left(Q_{X}\right)\right)
$$


for the canonical isomorphism. Let $\langle,\rangle_{X}$ be the Euler form for $\operatorname{rep}\left(Q_{X}\right)$. Since $F_{d_{X}}$ is exact, $\varphi_{X}, \varphi_{X}^{-1}$ are isometries, that is, for $a, b \in K_{0}\left(\operatorname{rep}\left(Q_{X}\right)\right)$, we have

$$
\langle a, b\rangle_{X}=\left\langle\varphi_{X}^{-1}(a), \varphi_{X}^{-1}(b)\right\rangle .
$$

Let us consider $P(X)$ the (possibly empty) set of all dimension vectors of the indecomposable projective representations of $\operatorname{rep}(Q)$ in ${ }^{\perp} X$. We let $H_{d_{X}}^{s s}$ denote the cone in $\mathbb{Q}^{n}$ generated by the elements of $-P(X) \cup\left\{d_{S_{1}^{X}}, \ldots, d_{S_{n-1}^{X}}\right\}$. As a first property of $H_{d_{X}}^{s s}$, we have the following.

Lemma 7.1. Let $\alpha$ be a real Schur root and let $d \in \mathbb{Z}^{n}$ with $d \in H_{\alpha}^{s s}$. Then $d_{+}, d_{-} \in H_{\alpha}^{s s}$.

Proof. Let $d_{1}, \ldots, d_{r}$ be the dimension vectors of the relative simple modules of ${ }^{\perp} M(\alpha)$. If $M(\alpha)$ is sincere, $d=d_{+}, d_{-}=0$ and there is nothing to prove. Otherwise, let $q_{1}, \ldots, q_{s}$ be the dimension vectors of the indecomposable projective representations that are in ${ }^{\perp} M(\alpha)$. Since $d \in H_{\alpha}^{s s}, d=\sum_{i=1}^{r} a_{i} d_{i}-\sum_{j=1}^{s} b_{j} q_{j}$ where the $a_{i}, b_{j}$ are non-negative integers. Now, consider the projective representation $P_{0}$ of dimension vector $\sum_{j=1}^{s} b_{j} q_{j}$ and consider $M_{0}=\bigoplus_{i=1}^{r} M\left(d_{i}\right)^{a_{i}}$, which is a representation in ${ }^{\perp} M(\alpha)$ of dimension vector $\sum_{i=1}^{r} a_{i} d_{i}$. If $\operatorname{Hom}\left(P_{0}, M_{0}\right)=0$, then $d_{+}=d_{M_{0}}, d_{-}=-d_{P_{0}}$ and we are done. Otherwise, let $f: P_{0} \rightarrow M_{0}$ be a nonzero homomorphism with kernel $P_{1}$ and cokernel $M_{1}$. Since $P_{0}, M_{0} \in{ }^{\perp} M(\alpha)$ and ${ }^{\perp} M(\alpha)$ is an abelian subcategory of $\operatorname{rep}(Q)$, we have $P_{1}, M_{1} \in{ }^{\perp} M(\alpha)$. Since $\operatorname{rep}(Q)$ is hereditary, $P_{1}$ is projective. Observe that $d=d_{M_{1}}-d_{P_{1}}$. If $\operatorname{Hom}\left(P_{1}, M_{1}\right)=0$, then $d_{+}=d_{M_{1}}, d_{-}=-d_{P_{1}}$ and we are done. Otherwise, we let the process goes by induction and we get a sequence of submodules $\cdots \subset P_{1} \subset P_{0}$, a sequence of quotients $M_{0} \rightarrow M_{1} \rightarrow \cdots$ such that $\operatorname{Hom}\left(P_{i}, M_{i}\right) \neq 0$ if and only if both $P_{i+1} \subset P_{i}$ and $M_{i} \rightarrow M_{i+1}$ are proper. Moreover, $d=d_{M_{i}}-d_{P_{i}}$ for all $i \geq 0$. Since $P_{0}$ is finite dimensional, there exists $t \geq 1$ such that $P_{t}=P_{j}$ whenever $j \geq t$. This gives $\operatorname{Hom}\left(P_{t}, M_{t}\right)=0$. Thus, $d_{+}=d_{M_{t}}, d_{-}=-d_{P_{t}}$. By construction, all $P_{i}$ and $M_{j}$ are in ${ }^{\perp} M(\alpha)$, which proves the lemma.

Now, if $Q$ is Euclidean and $\delta \in H_{d_{X}}^{s s}$, then $\varphi_{X}(\delta)$ is clearly the null root for the quiver $Q_{X}$ of ${ }^{\perp} X$. Observe that even when $Q$ is connected, $Q_{X}$ may be disconnected (a union of quivers of Dynkin type and at most one quiver of Euclidean type), thus, $\varphi_{X}(\delta)$ may be non-sincere.

Observe also that it is possible to have a dimension vector $f \in H_{d_{X}}^{s s}$ and a representation $M \in \operatorname{rep}(Q, f)$ with $M$ not isomorphic to any representation in ${ }^{\perp} X$. However, this does not happen for general representations. The notion of general representation used below was defined after Proposition 3.3 .

Lemma 7.2. Let $X$ be an exceptional representation and $f \in H_{d_{X}}^{s s}$ a dimension vector. Then there is a general representation of dimension vector $f$ in ${ }^{\perp} X$. Moreover, $M$ is a general representation of dimension vector $\varphi_{X}(f)$ in $\operatorname{rep}\left(Q_{X}\right)$ if and only if $G_{d_{X}}(M)$ is a general representation of dimension vector $f$ in ${ }^{\perp} X$. In particular, $\varphi_{X}, \varphi_{X}^{-1}$ preserve the canonical decomposition.

Proof. Since $f \in H_{d_{X}}^{s s}$, there exists a representation of dimension vector $f$ in ${ }^{\perp} X$. Let $\mathcal{U}$ be a non-empty open set of $\operatorname{rep}(Q, f)$ as in the definition of the canonical decomposition of $f$. A representation $N$ in $\operatorname{rep}(Q, f)$ lies in ${ }^{\perp} X$ if and only if the determinant $C^{N}(X)$ does not vanish. This defines a non-empty open set $\mathcal{U}^{\prime}$ in $\operatorname{rep}(Q, f)$. Being an affine space, $\operatorname{rep}(Q, f)$ is irreducible. The first statement 
follows from the fact the the intersection $\mathcal{U} \cap \mathcal{U}^{\prime}$ is non-empty and $\mathcal{U}$ only contains general representations. Being exact equivalences, it is clear that $F_{d_{X}}, G_{d_{X}}$ preserve the general representations.

If $X$ is exceptional, then the cd-fan for $Q$ restricts to a simplicial fan in $H_{d_{X}}^{s s} \cap$ $\left(\mathbb{Q}^{n}\right)_{+}$. From Lemma 7.2 this simplicial fan on $H_{d_{X}}^{s s} \cap\left(\mathbb{Q}^{n}\right)_{+}$corresponds, under $\varphi_{X}$, to the cd-fan for $\operatorname{rep}\left(Q_{X}\right)$. Let us note that it is not true that the cp-fan for $Q$ restricts to the cp-fan for $\operatorname{rep}\left(Q_{X}\right)$. The reason is that the relative projective objects in $\operatorname{rep}\left(Q_{X}\right)$ are not necessarily projective objects in $\operatorname{rep}(Q)$.

Recall, from Section 6, the definition of the set $R$ when $Q$ is Euclidean. To unify the notations, we set $R=\emptyset$ when $Q$ is Dynkin. For $Q$ Dynkin or Euclidean, let $\mathcal{J}=\mathcal{J}_{Q}=\left\{C_{I}\right\}_{I \in R} \cup\left\{H_{\alpha}^{s s}\right\}_{\alpha}$ where $\alpha$ runs through the set of all real Schur roots. Let $\mathcal{L}=\mathcal{L}_{Q}$ be the set of all subsets of $\mathbb{Q}^{n}$ that can be expressed as intersections of some subset of $\mathcal{J}$. This is called the intersection lattice of $\mathcal{J}$. For $L \in \mathcal{L}$, define the faces of $L$ to be the connected components of the set of points which are in $L$ but not in any smaller intersection. Define the faces of $\mathcal{L}$ to be the collection of all faces of all the elements of $\mathcal{L}$. Given $d \in \mathbb{Z}^{n}, \mathcal{J}_{d} \subseteq \mathcal{J}$ consists of all the elements in $\mathcal{J}$ containing $d$.

Lemma 7.3. Let $Q$ be a Dynkin or Euclidean quiver, $\alpha$ a real Schur root and $d \in \mathbb{Z}^{n}$. Then $d \in H_{\alpha}^{s s}$ if and only if $M(\alpha) \in \operatorname{rep}(Q)_{d}$.

Proof. Suppose that $M(\alpha) \in \operatorname{rep}(Q)_{d}$. Then from Corollary 3.2, there exist a positive integer $m$ and a representation $V$ supported over $Q_{\alpha}$ with $\left\langle d_{V},-\right\rangle \sim_{\alpha}$ $\langle m d,-\rangle$ and with $C^{V}(M(\alpha)) \neq 0$. Then $V \in{ }^{\perp} M(\alpha)$ and $d_{V} \in H_{\alpha}^{s s}$. If $M(\alpha)$ is sincere, then $d_{V}=m d$ and hence $d \in H_{\alpha}^{s s}$. Otherwise, $\left\langle d_{V}-d m,-\right\rangle \sim_{\alpha} 0$. There exists $f \in \mathbb{Z}^{n}$ having a support disjoint from $\alpha$ such that $\left\langle d_{V}-d m,-\right\rangle=f$, which gives $d_{V}-d m=f E^{-1}$, where $E$ is the matrix of the Euler form. But $f E^{-1}$ lies in the span of the dimension vectors of the projective representations whose tops are not supported over $Q_{\alpha}$. This gives $d \in H_{\alpha}^{s s}$. Conversely, assume $d \in H_{\alpha}^{s s}$. Write $d=d_{+}+d_{-}$, where $d_{+}$is a dimension vector and $-d_{-}$is the dimension vector of a projective representation such that $\left\langle d_{-}, d_{+}\right\rangle=0$. By Lemma [7.1] we have $d_{+}, d_{-} \in H_{\alpha}^{s s}$. Then a general representation of $Q_{M(\alpha)}$ of dimension vector $\varphi_{M(\alpha)}\left(d_{+}\right)$is a general representation of $Q$ of dimension vector $d_{+}$, and this gives us a supply of representations of dimension vector $d_{+}$which lie in ${ }^{\perp} M(\alpha)$. Any such representation $V$ will satisfy $C^{V}(M(\alpha)) \neq 0$. Since $\langle d,-\rangle$ and $\left\langle d_{+},-\right\rangle$agree on the support of $M(\alpha)$, we see by Propositions 5.1 and 5.2 that $M(\alpha) \in \operatorname{rep}(Q)_{d}$.

We will now state the main theorem of this section, and prove it in the Dynkin case. The proof in the Euclidean case is similar but requires some further lemmas, so we defer it to the end of this section.

Theorem 7.4. Let $Q$ be a Dynkin or Euclidean quiver and $d_{1}, d_{2} \in \mathbb{Z}^{n}$. Then $d_{1}$ and $d_{2}$ are ss-equivalent if and only if $\mathcal{J}_{d_{1}}=\mathcal{J}_{d_{2}}$.

Proof of Theorem 7.4 in the Dynkin case. Let $Q$ be a Dynkin quiver. Suppose that $d_{1}$ and $d_{2}$ are ss-equivalent. By definition, $\operatorname{rep}(Q)_{d_{1}}=\operatorname{rep}(Q)_{d_{2}}$, and Lemma 7.3 characterizes $\mathcal{J}_{d_{1}}$ and $\mathcal{J}_{d_{2}}$ in terms of this subcategory, so they must be equal. (Since $Q$ is Dynkin, $\mathcal{J}$ consists only of cones of the form $H_{\alpha}^{s s}$.)

Conversely, suppose $\mathcal{J}_{d_{1}}=\mathcal{J}_{d_{2}}$. By Lemma 7.3, $\operatorname{rep}(Q)_{d_{1}}$ and $\operatorname{rep}(Q)_{d_{2}}$ contain the same exceptional representations. Since $Q$ is Dynkin, all indecomposables are exceptional, and thus $\operatorname{rep}(Q)_{d_{1}}=\operatorname{rep}(Q)_{d_{2}}$. 
Suppose now that $Q$ is Euclidean. For $I \in R$, we denote by $\mathcal{W}_{I}$ the abelian, extension-closed subcategory generated by the indecomposable representations of dimension vectors in $F_{I}$. There is a unique quasi-simple in each non-homogeneous tube not contained in $\mathcal{W}_{I}$.

An indecomposable representation lying in a non-homogeneous tube and having dimension vector the null root will be called a singular-isotropic representation. For each quasi-simple of a non-homogeneous tube, there is a unique corresponding singular-isotropic representation for which there is a monomorphism from the quasisimple to the singular-isotropic representation.

For $Q$ having more than 2 vertices and $I \in R$, define $Z_{I}$ to be the direct sum of the singular-isotropic representations corresponding to the quasi-simples not in $\mathcal{W}_{I}$. We have the following simple lemma:

Lemma 7.5. For $Q$ Euclidean and having more than 2 vertices, ${ }^{\perp} Z_{I}$ consists of the additive hull of $\mathcal{W}_{I}$ together with the homogeneous tubes.

Proof. Lemma 4.3 tells us that ${ }^{\perp} Z_{I}$ is contained in $\mathcal{R}$ eg. It is clear that the homogeneous tubes lie in ${ }^{\perp} Z_{I}$. The rest is just a simple check within each of the non-homogeneous tubes.

We can now prove an analogue of Lemma 7.3 for the $C_{I}$.

Lemma 7.6. Let $Q$ be a Euclidean quiver having $n$ vertices and let $d \in \mathbb{Z}^{n}$. If $n>2$, then for $I \in R$, we have $d \in C_{I}$ if and only if $Z_{I} \in \operatorname{rep}(Q)_{d}$. If $n=2$, then $d \in C_{I}=H_{\delta}^{s s}$ if and only if $\operatorname{rep}(Q)_{d}=\mathcal{R}$ eg.

Proof. The case $n=2$ is trivial. If $Z_{I} \in \operatorname{rep}(Q)_{d}$, since it is sincere, then there is some representation $V$ with dimension vector $m d$ such that $C^{V}\left(Z_{I}\right) \neq 0$, and indeed, a Zariski open subset of the representations with dimension $m d$ have this property. By Lemma 7.5, this implies that the canonical decomposition of $m d$ must contain Schur roots from $\mathcal{W}_{I}$ together with some multiple of $\delta$, and this implies that $d$ lies in $C_{I}$.

Conversely, if $d$ lies in $C_{I}$, then the canonical decomposition of $d$ consists of Schur roots from $\mathcal{W}_{I}$, together with some non-negative multiple of $\delta$. Lemma 7.5 implies that there exist representations of dimension vector $d$ which lie in ${ }^{\perp} Z_{I}$, so $Z_{I} \in \operatorname{rep}(Q)_{d}$.

Lemmas 7.3 and 7.6 tell us that $\mathcal{J}_{d}$ contains enough information to determine exactly which exceptional or singular-isotropic indecomposable representations lie in $\operatorname{rep}(Q)_{d}$. We will use the following lemma to show that this is enough information to reconstruct $\operatorname{rep}(Q)_{d}$.

Lemma 7.7. Let $Q$ be Euclidean and let $\mathcal{A}, \mathcal{B}$ be extension-closed abelian subcategories of $\operatorname{rep}(Q)$. Suppose $\mathcal{A}$ and $\mathcal{B}$ have the same intersection with the exceptional representations and the indecomposables whose dimension vector is the null root. Then $\mathcal{A}=\mathcal{B}$.

Proof. Suppose there is some object $X \in \mathcal{A}$. We want to show that $X \in \mathcal{B}$. We may assume that $X$ is indecomposable. If $X$ is exceptional or $X$ is at the bottom of a homogeneous tube, then $X \in \mathcal{B}$ by assumption, so assume otherwise.

Suppose that the dimension vector of $X$ is $m \delta$ for some $m>1$. We claim that $X$ admits a filtration by representations each of whose dimension vectors is the null root, and each of which lies in $\mathcal{A}$. The proof is by induction on $m$. Suppose that 
the tube containing $X$ has width $r$. There is a path of length $(m-1) r$ consisting of irreducible epimorphisms from $X$ to a singular-isotropic representation $Y$. There is also a path of length $(m-1) r$ consisting of irreducible monomorphisms from $X$ to a representation $Z$ of dimension vector $(2 m-1) \delta$. This yields a monomorphism $X \rightarrow Y \oplus Z$ whose cokernel is clearly $X$. Hence, there is a short exact sequence

$$
0 \rightarrow X \rightarrow Y \oplus Z \rightarrow X \rightarrow 0 \text {. }
$$

It follows that $Y \in \mathcal{A}$. Now, let $K$ be the kernel of the surjection from $X$ onto $Y$. Since $\mathcal{A}$ is abelian, $K$ also lies in $\mathcal{A}$, and its dimension vector is $(m-1) \delta$. By induction, it admits a filtration as desired, and therefore so does $X$. Now since each of the terms of the filtration lies in $\mathcal{A}$ and has dimension vector $\delta$, they each also lie in $\mathcal{B}$, and therefore $X \in \mathcal{B}$.

Finally, suppose that the dimension vector of $X$ is of the form $m \delta+\alpha$, where $\alpha$ is a real Schur root. Similarly to the previous situation, there is an extension of $X$ by $X$ which has $M(\alpha)$ as an indecomposable summand, so $M(\alpha)$ lies in $\mathcal{A}$, and so does the kernel $K$ of the map from $X$ to $M(\alpha)$. The dimension vector of $K$ is $m \delta$, so by what we have already established, it lies in $\mathcal{B}$, and so does $M(\alpha)$, so $X$ does as well.

Proof of Theorem 7.4 in the Euclidean case. If $d_{1}$ and $d_{2}$ are ss-equivalent, then, by definition, $\operatorname{rep}(Q)_{d_{1}}=\operatorname{rep}(Q)_{d_{2}}$, and now Lemmas 7.3 and 7.6 characterize $\mathcal{J}_{d_{1}}$ and $\mathcal{J}_{d_{2}}$ in terms of this subcategory, so they are equal.

Conversely, if $\mathcal{J}_{d_{1}}=\mathcal{J}_{d_{2}}$, then by Lemmas 7.3 and 7.6 $\operatorname{rep}(Q)_{d_{1}}$ and $\operatorname{rep}(Q)_{d_{2}}$ agree as to their intersection with exceptional representations and singular-isotropic representations. In order to apply Lemma 7.7, we also need to check their intersections with the quasi-simples of homogeneous tubes. If $\mathcal{J}_{d_{1}}$ and $\mathcal{J}_{d_{2}}$ do not include any $C_{I}$, then neither $d_{i}$ lies on $H_{\delta}^{s s}$, so neither $\operatorname{rep}(Q)_{d_{1}}$ nor $\operatorname{rep}(Q)_{d_{2}}$ contains any homogeneous tubes by Lemma 4.3 and Proposition 5.2. On the other hand, if $\mathcal{J}_{d_{1}}$ and $\mathcal{J}_{d_{2}}$ do contain some $C_{I}$, then $d_{1}$ and $d_{2}$ both lie on the regular hyperplane, and thus both $\operatorname{rep}(Q)_{d_{1}}$ and $\operatorname{rep}(Q)_{d_{2}}$ contain all the homogeneous tubes by Lemmas 4.3 and 5.3 and Proposition 5.4. In either case, we see that $\operatorname{rep}(Q)_{d_{1}}$ and $\operatorname{rep}(Q)_{d_{2}}$ agree as to their intersections with quasi-simples from homogeneous tubes as well, and therefore Lemma 7.7 applies to tell us that $\operatorname{rep}(Q)_{d_{1}}=\operatorname{rep}(Q)_{d_{2}}$.

\section{Properties of the CP-FAN AND Proof of TheOrem 8.3}

In this section, we prove Theorem 8.3, describing when two elements of $\mathbb{Z}^{n}$ have canonical presentations whose summands correspond to the same rays in the $\mathrm{cp}-$ fan. Recall that since $Q$ is Dynkin or Euclidean, the rays of the cp-fan correspond bijectively to the summands occurring in cp-presentations. All the quivers in this section are Dynkin or Euclidean.

In the sequel, an additive $k$-category $\mathcal{C}$ will be called representation-finite, or of finite type, if it contains finitely many indecomposable objects, up to isomorphism. In the Euclidean case, all the categories $\mathcal{W}_{I}$ are representation-finite, abelian, extension-closed subcategories of $\mathcal{R}$ eg. The following shows that the $\mathcal{W}_{I}$ are the maximal such subcategories.

Lemma 8.1. Let $Q$ be Euclidean and $\mathcal{C}$ be an abelian, extension-closed subcategory of $\operatorname{rep}(Q)$ contained in $\mathcal{R}$ eg. Then $\mathcal{C}$ is representation-finite if and only if $\mathcal{C}$ is contained in some $\mathcal{W}_{I}$. 
Proof. We only need to prove the necessity. Suppose that $\mathcal{C}$ is representation-finite but not included in any of the $\mathcal{W}_{I}$. If $\mathcal{C}$ contains an indecomposable non-exceptional representation $X$, then $\mathcal{C}$ clearly contains infinitely many indecomposable representations lying in the same component as $X$ of the Auslander-Reiten quiver of $\operatorname{rep}(Q)$, so suppose this does not hold. Since $\mathcal{C}$ is not contained in any $\mathcal{W}_{I}$, there exists a nonhomogeneous tube $\mathcal{T}$, say of rank $r$, for which $\mathcal{C} \cap \mathcal{T}$ is not contained in any abelian, extension-closed subcategory of $\mathcal{T}$ generated by $r-1$ quasi-simple representations. Let $X$ be an exceptional representation in $\mathcal{C} \cap \mathcal{T}$ of largest quasi-length. Then $X$ is such that $\operatorname{Ext}^{1}(X, \mathcal{C} \cap \mathcal{T})=\operatorname{Ext}^{1}(\mathcal{C} \cap \mathcal{T}, X)=0$ since otherwise, this would provide a representation in $\mathcal{C} \cap \mathcal{T}$ with quasi-length larger than that of $X$. Suppose that the quasi-simple composition factors of $X$ are $S, \tau S, \ldots, \tau^{m} S, 0 \leq m<r$. Since $X$ is exceptional, $\tau^{m+1} S \not S$ and $\tau^{m+1} S$ is not a quasi-simple composition factor of $X$ and any $Y \in \mathcal{C} \cap \mathcal{T}$ having $\tau^{m+1} S$ as a quasi-simple composition factor is such that $\operatorname{Ext}(X, Y) \cong D \operatorname{Hom}(Y, \tau X) \neq 0$, which is impossible. Hence, $\mathcal{C} \cap \mathcal{T}$ is contained in the extension-closed abelian subcategory of $\mathcal{T}$ generated by all the quasi-simple representations except $\tau^{m+1} S$. This is a contradiction.

We say that two elements $d_{1}, d_{2}$ in $\mathbb{Z}^{n}$ are cp-equivalent if the canonical presentations of $d_{1}, d_{2}$ involve the same summands, up to multiplicity.

Example 8.2. Let $Q$ be the quiver $2 \longrightarrow 1$. The indecomposable representations, up to isomorphism, are the simple representations $S_{1}, S_{2}$ at vertices 1,2 , respectively and the projective-injective representation $P_{2}$. The canonical presentation of $(1,2)$ is $(1,1)+(0,1)$ and that of $(2,3)$ is $2(1,1)+(0,1)$. Therefore, $(1,2),(2,3)$ are cp-equivalent. The canonical presentation of $(-2,-3)$ is $3(-1,-1)+1(1,0)$. So $(-2,-3)$ is cp-equivalent to $(-b,-a)$ for $a, b$ positive integers with $b<a$.

The following result gives an explicit description of when two elements are cpequivalent.

Theorem 8.3. Let $Q$ be a Dynkin or Euclidean quiver. Two elements $d_{1}, d_{2}$ in $\mathbb{Z}^{n}$ are cp-equivalent if and only if they lie in the same face of $\mathcal{L}_{Q}$.

Proof. Let $d_{1}, d_{2} \in \mathbb{Z}^{n}$. Suppose that $d_{1}, d_{2}$ are cp-equivalent. Then any nonnegative integer linear combination of $d_{1}$ and $d_{2}$ will also be cp-equivalent to both of them, by Lemma 3.8. If $d_{1}, d_{2}$ do not lie on the same face of $\mathcal{L}_{Q}$, either $\{J \in$ $\left.\mathcal{J}_{Q} \mid d_{1} \in J\right\} \neq\left\{J \in \mathcal{J}_{Q} \mid d_{2} \in J\right\}$ or $\left\{J \in \mathcal{J}_{Q} \mid d_{1} \in J\right\}=\left\{J \in \mathcal{J}_{Q} \mid d_{2} \in J\right\}$ but there exists $H \in \mathcal{J}_{Q}$ such that $\left(\cap_{d_{2} \in J} J\right) \backslash H$ consists of multiple components, with $d_{1}$ and $d_{2}$ in different components. In both cases, there is a vector $d_{3}$ which lies on the straight line joining $d_{1}, d_{2}$ and $H \in \mathcal{J}_{Q}$ such that $d_{3} \in H$ and at least one of $d_{1}, d_{2}$ does not lie on $H$. Without loss of generality, suppose that $d_{1} \notin H$. As we have already remarked, $d_{3}$ must be cp-equivalent to $d_{1}$, and $\left(d_{3}\right)_{+}$must be cp-equivalent to $\left(d_{1}\right)_{+}$. Also, since $d_{3}$ lies on $H$, by Lemma 7.1 $\left(d_{3}\right)_{+}$does.

If $H=H_{\alpha}^{s s}$ for a real Schur root $\alpha$, then there is a general representation $N$ of dimension vector $\left(d_{3}\right)_{+}$such that $N \in{ }^{\perp} M(\alpha)$ by Lemma 7.3. But there exists a general representation $N^{\prime}$ (built from the indecomposable direct summands of $N$ ) of dimension vector $\left(d_{1}\right)_{+}$that has the indecomposable summands of the same dimensions (though different multiplicities), so $N^{\prime} \in{ }^{\perp} M(\alpha)$, showing that $\left(d_{1}\right)_{+} \in H_{\alpha}^{s s}$ by Lemma 7.3. Now, the fact that $\left(d_{3}\right)_{-} \in H_{\alpha}^{s s}$ clearly implies that $\left(d_{1}\right)_{-} \in H_{\alpha}^{s s}$. This gives, by the convexity of $H_{\alpha}^{s s}$, that $d_{1} \in H_{\alpha}^{s s}$, a contradiction.

Similarly, if $\left|Q_{0}\right|>2$ and $H=C_{I}$ for some $I \in R$, then $d_{3}$ is a dimension vector and we deduce that a general representation $N$ of dimension vector $d_{3}$ lies 
in ${ }^{\perp} Z_{I}$ by Lemma 7.6. As argued above, there will be a general representation $N^{\prime}$ of dimension vector $d_{1}$ which lies in ${ }^{\perp} Z_{I}$, showing that $d_{1} \in C_{I}$, a contradiction. If $\left|Q_{0}\right|=2$ and $H=C_{\emptyset}=H_{\delta}^{s s}$, then by cp-equivalence, both $d_{1}, d_{3}$ are multiples of $\delta$ and it is clear that $d_{1} \in H$, a contradiction. This establishes one direction.

Suppose now that $d_{1}, d_{2}$ lie in the same face of $\mathcal{L}_{Q}$. Suppose further that the canonical presentation of $d_{1}$ involves distinct vectors $f_{1}, \ldots, f_{r}$, where $f_{i}$ is either a Schur root or the negative of the dimension vector of a projective indecomposable representation. By Lemma 3.8, any positive linear combination of $f_{1}, \ldots, f_{r}$ will have the same summands appearing in its canonical presentation as for $d_{1}$. Thus, we will be done if we can show that the fact that $d_{2}$ lies in the same face of $\mathcal{L}_{Q}$, implies that $d_{2}$ lies in the cone generated by the $f_{i}$. Since the $f_{i}$ are linearly independent, the boundary facets of this cone are spanned by any $r-1$ of the $f_{i}$. We will show that for any $1 \leq i \leq r$, we have that $\left\{f_{1}, \ldots, f_{i-1}, f_{i+1}, \ldots, f_{r}\right\}$ lies on some $H \in \mathcal{J}$ with $f_{i} \notin H$. The rest of the proof is devoted to proving the claim, where it suffices to consider one particular value of $i$. So let us consider $i=r$.

Suppose first that $\left\{f_{1}, \ldots, f_{r}\right\}$ does not contain the null root. For each $i$, let $f_{i}^{\prime}=$ $f_{i}$ if $f_{i}$ is a Schur root and $f_{i}^{\prime}=-f_{i}$ otherwise. The representations $M\left(f_{i}^{\prime}\right)$ can be ordered into an exceptional sequence: those corresponding to positive roots can be so ordered because they form a partial tilting object, and then those corresponding to negative roots can be put at the front. Now remove $M\left(f_{r}^{\prime}\right)$. The resulting exceptional sequence can be completed to a full exceptional sequence by adding some terms $X_{r}, \ldots, X_{n}$ to the end. If we re-insert $M\left(f_{r}^{\prime}\right)$ into the sequence at the place where it was before, the resulting sequence is too long to be exceptional, so there is some $X_{k}$ with $r \leq k \leq n$ such that $M\left(f_{r}^{\prime}\right) \notin{ }^{\perp} X_{k}$, while $M\left(f_{j}^{\prime}\right) \in{ }^{\perp} X_{k}$ for $1 \leq j<r$, by virtue of the exceptional sequence property. Therefore, $f_{r}^{\prime} \notin H_{d_{X_{k}}}^{s s}$ and $f_{j}^{\prime} \in H_{d_{X_{k}}}^{s s}$ for $1 \leq j<r$. It is clear that for $1 \leq j \leq r, f_{j} \in H_{d_{X_{k}}}^{s s}$ if and only if $f_{j}^{\prime} \in H_{d_{X_{k}}}^{s s}$. This shows that $H_{d_{X_{k}}}^{s s}$ is a hyperplane of the desired type. This case has now been dealt with, which completes the proof of the second direction in the Dynkin setting.

Next, assume $Q$ is Euclidean and suppose that $f_{r}=\delta$. In this case, all the other $f_{i}$ are dimension vectors. Moreover $\bigoplus_{1 \leq j<r} M\left(f_{j}\right)$ forms a regular partial tilting object by Lemma 4.3, and its summands can be ordered into an exceptional sequence. Complete this to an exceptional sequence by adding some terms $X_{r}, \ldots, X_{n}$. Since a full exceptional sequence cannot consist entirely of regular objects, there is some $X_{k}$ which is not regular. Now $H_{d_{X_{k}}}^{s s}$ contains $f_{1}, \ldots, f_{r-1}$, but by Lemma 4.3 it does not contain $f_{r}=\delta$. Thus $H_{d_{X_{k}}}^{s s}$ has the desired properties.

Finally, suppose that some $f_{j}=\delta$ for some $1 \leq j \leq r-1$. For convenience, let $f_{r-1}=\delta$. Then $M\left(f_{1}\right), \ldots, M\left(f_{r-2}\right)$ and $M\left(f_{r}\right)$ are the summands of a partial tilting object and can be ordered into an exceptional sequence which is contained in the regular component. Since they can be ordered into an exceptional sequence contained in the regular component, they generate an abelian subcategory of finite representation type, which is therefore contained in some wing $\mathcal{W}_{I}$ by Lemma 8.1 Delete $M\left(f_{r}\right)$ from the sequence, and then complete it to an exceptional sequence which is full inside $\mathcal{W}_{I}$, by adding some terms $X_{r-1}, \ldots, X_{n-2}$. Since $M\left(f_{r}\right)$ cannot be added back into the sequence, we see that there is some $X_{k}$ such that $M\left(f_{r}\right)$ is not in ${ }^{\perp} X_{k}$, so $f_{r} \notin H_{d_{X_{k}}}^{s s}$, while by the exceptional sequence property, $f_{t} \in H_{d_{X_{k}}}^{s s}$ for $1 \leq t \leq r-2$, and $f_{r-1} \in H_{d_{X_{k}}}^{s s}$ because $f_{r-1}=\delta$ and $X_{k}$ is regular. 


\section{Thick subcategories}

This section is devoted to proving some facts concerning extension-closed abelian subcategories of $\operatorname{rep}(Q)$. For part of the section, we assume only that $Q$ is acyclic; later, we assume that $Q$ is Euclidean.

We begin in an even more general setting. Let $\mathcal{H}$ be a hereditary abelian $k$ category. A (full) subcategory $\mathcal{A}$ of $\mathcal{H}$ is thick if it is closed under direct summands and whenever we have a short exact sequence with two terms in $\mathcal{A}$, then the third term also lies in $\mathcal{A}$. We start with the following result which is well known; see for example [13, Theorem 3.3.1]. We include a proof for the convenience of the reader.

Proposition 9.1. Let $\mathcal{H}$ be a hereditary abelian $k$-category with a full subcategory $\mathcal{A}$. Then $\mathcal{A}$ is extension-closed abelian if and only if it is thick.

Proof. The necessity follows trivially. Suppose that $\mathcal{A}$ is thick. We need to show that $\mathcal{A}$ has kernels and cokernels. Let $f: A \rightarrow B$ be a morphism in $\mathcal{A}$ with kernel $u: K \rightarrow A$, cokernel $v: B \rightarrow C$ and coimage $g: A \rightarrow E$. Since $\mathcal{H}$ is hereditary and we have a monomorphism $g^{\prime}: E \cong \operatorname{im}(f) \rightarrow B$, we have a surjective map $\operatorname{Ext}^{1}\left(g^{\prime}, K\right): \operatorname{Ext}^{1}(B, K) \rightarrow \operatorname{Ext}^{1}(E, K)$. The short exact sequence $0 \rightarrow K \rightarrow A \rightarrow E \rightarrow 0$ is an element in $\operatorname{Ext}^{1}(E, K)$ and hence is the image of an element in $\operatorname{Ext}^{1}(B, K)$. We have a pullback diagram

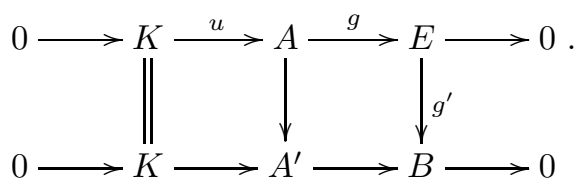

This gives rise to a short exact sequence

$$
0 \rightarrow A \rightarrow A^{\prime} \oplus E \rightarrow B \rightarrow 0 \text {. }
$$

Since $A, B \in \mathcal{A}$ and $\mathcal{A}$ is thick, we get $E \in \mathcal{A}$. Hence, $K, C \in \mathcal{A}$.

Thanks to $\mathcal{H}$ being hereditary, the bounded derived category of $\mathcal{H}$, written as $D^{b}(\mathcal{H})$, is easy to describe. Recall that a stalk complex in $D^{b}(\mathcal{H})$ is a complex concentrated in one degree, that is, a complex of the form $X[i]$ for an object $X$ in $\mathcal{H}$. From [23], every object in $D^{b}(\mathcal{H})$ is a finite direct sum of stalk complexes. Observe also that given two objects $X, Y \in \mathcal{H}$, the condition $\operatorname{Hom}_{D^{b}(\mathcal{H})}(X, Y[i]) \neq 0$ implies either $i=0$ or $i=1$. A full subcategory $\mathcal{A}$ of $D^{b}(\mathcal{H})$ is thick if it closed under direct summands and whenever we have a map $U \rightarrow V$ in $\mathcal{A}$, then the distinguished triangle

$$
W \rightarrow U \rightarrow V \rightarrow W[1]
$$

lies in $\mathcal{A}$. In particular, a thick subcategory is closed under shifts and is a triangulated subcategory of $D^{b}(\mathcal{H})$. Given a thick subcategory $\mathcal{A}$ of $D^{b}(\mathcal{H})$, we denote by $H^{0}(\mathcal{A})$ the category $\mathcal{H} \cap \mathcal{A}$, that is, the complexes $C$ in $\mathcal{A}$ for which $H^{i}(C)=0$ for all $i \neq 0$. The category $H^{0}(\mathcal{A})$ is abelian and extension-closed, and hence is a thick subcategory of $\mathcal{H}$. Observe also that it is the heart of the $t$-structure on $\mathcal{A}$ coming from the canonical $t$-structure on $D^{b}(\mathcal{H})$; see 16 . It is easily seen that $\mathcal{A}$ is triangle-equivalent to the bounded derived category of $H^{0}(\mathcal{A})$.

Given a family of objects $E$ in $D^{b}(\mathcal{H})$, we write $\mathcal{D}(E)$ for the thick subcategory of $D^{b}(\mathcal{H})$ generated by the objects in $E$. We define $\mathcal{D}(E)^{\perp}$ (resp. ${ }^{\perp} \mathcal{D}(E)$ ) to be the full subcategory of $D^{b}(\mathcal{H})$ generated by the objects $Y$ with $\operatorname{Hom}(X, Y[i])=0$ 
(resp. $\operatorname{Hom}(Y, X[i])=0$ ) for all $X \in E$ and all $i \in \mathbb{Z}$. Observe that if $E \subseteq \mathcal{H}$, $\mathcal{C}(E)=H^{0}(\mathcal{D}(E))$ and $H^{0}\left(\mathcal{D}(E)^{\perp}\right)=\mathcal{C}(E)^{\perp}$. As on the level of abelian categories, an indecomposable object $X$ in $D^{b}(\mathcal{H})$ is exceptional if $\operatorname{Hom}(X, X[i])=0$ for all nonzero integers $i$ and $\operatorname{End}(X) \cong k$. An exceptional sequence $E=\left(X_{1}, X_{2}, \ldots, X_{r}\right)$ in $D^{b}(\mathcal{H})$ is a sequence of exceptional objects for which

$$
\operatorname{Hom}\left(X_{i}, X_{j}[l]\right)=0 \text { for all } l \text { and } 1 \leq i<j \leq r .
$$

Any exceptional sequence in $\mathcal{H}$ is also an exceptional sequence in $D^{b}(\mathcal{H})$. Conversely, if $E=\left(X_{1}, X_{2}, \ldots, X_{r}\right)$ is exceptional in $D^{b}(\mathcal{H})$, then there exist integers $i_{1}, i_{2}, \ldots, i_{r}$ for which $H^{0}(E):=\left(X_{1}\left[i_{1}\right], \ldots, X_{r}\left[i_{r}\right]\right)$ is an exceptional sequence in $\mathcal{H}$.

Let us introduce more terminology. Let $\mathcal{E}$ be any $k$-linear category. A full subcategory $\mathcal{F}$ of $\mathcal{E}$ is said to be contravariantly finite if for any object $E$ in $\mathcal{E}$, there exists a morphism $f: F \rightarrow E$ with $F \in \mathcal{F}$ such that $\operatorname{Hom}\left(F^{\prime}, f\right)$ is surjective for any $F^{\prime} \in \mathcal{F}$. Such a morphism $f$ is called a right $\mathcal{F}$-approximation of $E$. The following result is stated at the derived category level but is also true at the abelian category level.

Lemma 9.2. Suppose that $\mathcal{H}$ is Hom-finite. Let $E=\left(X_{1}, X_{2}, \ldots, X_{r}\right)$ be an exceptional sequence in $D^{b}(\mathcal{H})$. Then $\mathcal{D}(E)$ is contravariantly finite in $D^{b}(\mathcal{H})$.

Proof. We proceed by induction on $r$. If $r=1$, then $\mathcal{D}(E)$ is the additive category containing the shifts of $X_{1}$. This is clearly contravariantly finite in $D^{b}(\mathcal{H})$. Suppose now that $r>1$. We have that $E^{\prime}=\left(X_{1}, \ldots, X_{r-1}\right)$ is an exceptional sequence with $\mathcal{D}\left(E^{\prime}\right)$ contravariantly finite. Let $M$ be any object in $D^{b}(\mathcal{H})$ with $f: C \rightarrow M$ a right $\mathcal{D}\left(E^{\prime}\right)$-approximation of $M$. Since $\mathcal{H}$ is abelian and Hom-finite, $\mathcal{H}$, and hence $D^{b}(\mathcal{H})$, is Krull-Schmidt. Therefore, we may assume that $f$ is right-minimal, in the sense that if $\varphi: C \rightarrow C$ is such that $f=f \varphi$, then $\varphi$ is an isomorphism. Now, we have a triangle

$$
N \stackrel{g}{\longrightarrow} C \stackrel{f}{\longrightarrow} M \longrightarrow N[1] .
$$

We claim that $N$ lies in $\mathcal{D}\left(E^{\prime}\right)^{\perp}$. Let $u: B \rightarrow N$ be a morphism with $B \in \mathcal{D}\left(E^{\prime}\right)$. By the octahedral axiom, we have a commutative diagram

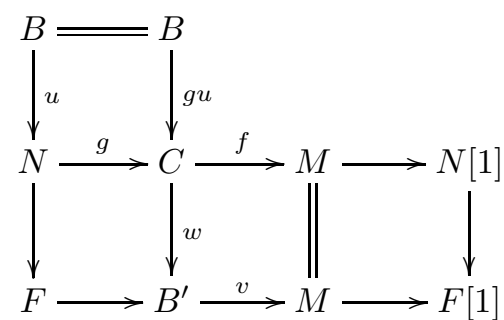

where $B^{\prime}$ is an object in $\mathcal{D}\left(E^{\prime}\right)$ and all rows and columns are distinguished triangles. Since $f$ is a right $\mathcal{D}\left(E^{\prime}\right)$-approximation of $M, v$ factors through $f$ and hence, there exists $w^{\prime}: B^{\prime} \rightarrow C$ with $f=f w^{\prime} w$. Hence, since $f$ is right minimal, we get that $w$ is a section, which means that $g u=0$. Hence, $u$ factors through $M[-1]$. But since $B$ is in $\mathcal{D}\left(E^{\prime}\right)$ and $f[-1]$ is a right $\mathcal{D}\left(E^{\prime}\right)$-approximation of $M[-1], u$ factors through $f[-1]$ and therefore, $u=0$. This proves that $N$ lies in $\mathcal{D}\left(E^{\prime}\right)^{\perp}$.

Now, let $\mathcal{X}$ be the thick subcategory of $D^{b}(\mathcal{H})$ generated by $X_{r}$, which is contravariantly finite in $D^{b}(\mathcal{H})$ using the base case $r=1$. Let $h: X \rightarrow N$ be a minimal 
right $\mathcal{X}$-approximation of $N$. The octahedral axiom gives a commutative diagram

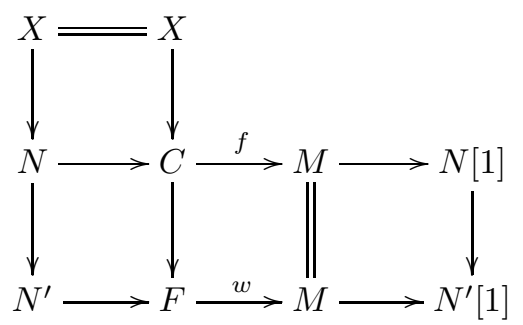

where $N^{\prime}$ is an object in $\mathcal{D}\left(E^{\prime}\right)^{\perp} \cap \mathcal{X}^{\perp}=\mathcal{D}(E)^{\perp}$ and all rows and columns are distinguished triangles. It is easily seen that the morphism $w: F \rightarrow M$ is a right $\mathcal{D}(E)$-approximation of $M$.

Proposition 9.3. Let $\mathcal{H}$ be a Hom-finite hereditary abelian $k$-category with a nonfull exceptional sequence $E=\left(X_{1}, \ldots, X_{r}\right)$ in $D^{b}(\mathcal{H})$ and such that all indecomposable objects in $\mathcal{D}(E)^{\perp}$ are exceptional. Then $E$ can be extended to an exceptional sequence $E=\left(X_{1}, \ldots, X_{r}, X_{r+1}\right)$.

Proof. We only need to prove that the category $\mathcal{D}(E)^{\perp}$ is nonzero. By the assumption, there is an indecomposable object $M$ which is not in $\mathcal{D}(E)$. By Lemma 9.2 . we have a triangle

$$
N \stackrel{g}{\longrightarrow} C \stackrel{f}{\longrightarrow} M \longrightarrow N[1]
$$

where $f$ is a right $\mathcal{D}(E)$-approximation of $M$. Then $N$ is nonzero in $\mathcal{D}(E)^{\perp}$ and has an exceptional object $X_{r+1}$ as a direct summand. This yields an exceptional sequence $E=\left(X_{1}, \ldots, X_{r}, X_{r+1}\right)$.

In the rest of this section, we specialize to $\mathcal{H}=\operatorname{rep}(Q)$ for an acyclic quiver $Q$ with $n$ vertices. Given two thick subcategories $\mathcal{C}_{1}, \mathcal{C}_{2}$ of $\operatorname{rep}(Q)$, we call the pair $\left(\mathcal{C}_{1}, \mathcal{C}_{2}\right)$ a semi-orthogonal pair if $\mathcal{C}_{2}=\mathcal{C}_{1}^{\perp}$. In order to deal with such pair, we need the following easy observation.

Lemma 9.4. Let $\mathcal{C}$ be a finitely generated abelian, extension-closed subcategory of $\operatorname{rep}(Q)$. Then ${ }^{\perp}\left(\mathcal{C}^{\perp}\right)=\mathcal{C}$.

Proof. The category $\mathcal{C}$ is generated by an exceptional sequence $\left(X_{1}, X_{2}, \ldots, X_{r}\right)$ that can be completed to a full exceptional sequence $\left(X_{1}, \ldots, X_{r}, \ldots, X_{n}\right)$. Then $\mathcal{C}^{\perp}$ is generated by $X_{r+1}, \ldots, X_{n}$. It is clear that $\mathcal{C} \subseteq \bigcap_{i=r+1}^{n}{ }^{\perp} X_{i}$ and that $\bigcap_{i=r+1}^{n}{ }^{\perp} X_{i}$ is equivalent to the category of representations of a quiver with $n-$ $(n-r)=r$ vertices. If equality does not hold, then $\left(X_{1}, \ldots, X_{r}\right)$ is not full in $\bigcap_{i=r+1}^{n}{ }^{\perp} X_{i}$, which is a contradiction.

Given a full exceptional sequence $E=\left(X_{1}, X_{2}, \ldots, X_{n}\right)$ in $\operatorname{rep}(Q)$ and an integer $0 \leq s \leq n$, there is a semi-orthogonal pair

$$
\mathcal{P}(E, s):=\left(\mathcal{C}\left(E_{\leq s}\right), \mathcal{C}\left(E_{>s}\right)\right),
$$

where $E_{\leq s}=\left(X_{1}, \ldots, X_{s}\right)$ and $E_{>s}=\left(X_{s+1}, \ldots, X_{n}\right)$. We set $E_{\leq 0}=\emptyset$ and $E_{>n}=\emptyset$. It is clear that $\mathcal{C}\left(E_{>s}\right) \subseteq \mathcal{C}\left(E_{\leq s}\right)^{\perp}$. Moreover, $E_{>s}$ is an exceptional sequence in $\mathcal{C}\left(E_{\leq s}\right)^{\perp}$, which is equivalent to $\operatorname{rep}\left(Q^{\prime}\right)$ for some acyclic quiver $Q^{\prime}$ with $n-s$ vertices. Since any exceptional sequence in $\operatorname{rep}\left(Q^{\prime}\right)$ can be completed to a full exceptional sequence, see [9], and $E$ is full, we see that $\mathcal{C}\left(E_{>s}\right)=\mathcal{C}\left(E_{\leq s}\right)^{\perp}$. 
An exceptional pair of the form $\mathcal{P}(E, s)$ where $E=\left(X_{1}, X_{2}, \ldots, X_{n}\right)$ is a full exceptional sequence and $1 \leq s \leq n$ will be called an exceptional semi-orthogonal pair.

Lemma 9.5. Let $Q$ be any acyclic quiver and let $\mathcal{C}$ be any thick subcategory of $\operatorname{rep}(Q)$ which is representation-finite. Then $\mathcal{C}$ is equivalent to $\operatorname{rep}\left(Q^{\prime}\right)$ for some (possibly disconnected) Dynkin quiver $Q^{\prime}$.

Proof. We prove the statement by induction on the number $r$ of non-isomorphic indecomposable objects in $\mathcal{C}$. If $r \leq 1$, the result is clear. So assume $r \geq 2$. Let $X_{0}$ be an indecomposable representation in $\mathcal{C}$ of least dimension over $k$. Since $\mathcal{C}$ is thick, we get $\operatorname{End}\left(X_{0}\right) \cong k$. Using the same argument as in the proof of the lemma in [16. p. 166], we get $\operatorname{Ext}^{1}\left(X_{0}, X_{0}\right)=0$. Hence, $X_{0}$ is exceptional. Now, in $\mathcal{C}, X_{0}^{\perp}$ is thick and representation-finite with fewer non-isomorphic indecomposable objects. By induction, $X_{0}^{\perp}$ is equivalent to $\operatorname{rep}\left(Q^{\prime \prime}\right)$ where $Q^{\prime \prime}$ is a (possibly disconnected) Dynkin quiver. Now, from Proposition 4.4 $X_{0}^{\perp}$ is given by an exceptional sequence $\left(X_{1}, \ldots, X_{m}\right)$. Let $E$ be the exceptional sequence $\left(X_{0}, X_{1}, \ldots, X_{m}\right)$ in $\mathcal{C}$. It is clear that $\mathcal{C}$ is a hom-finite hereditary abelian $k$-category, and $E$ is also an exceptional sequence in the bounded derived category $D^{b}(\mathcal{C})$ of $\mathcal{C}$. By Proposition 9.3, since $\mathcal{D}(E)^{\perp}$ is zero, we get that $E$ is full. By Proposition $4.4 \mathcal{C}$ is equivalent to $\operatorname{rep}\left(Q^{\prime}\right)$ for some acyclic quiver $Q^{\prime}$. Since $\mathcal{C}$ is representation-finite, $Q^{\prime}$ is a union of quivers of Dynkin type.

The preceding lemma yields the following: for an acyclic quiver $Q$, any thick subcategory of $\operatorname{rep}(Q)$ which is representation-finite is generated by an exceptional sequence.

A connecting component of $D^{b}(\operatorname{rep}(Q))$ is a connected component of the AuslanderReiten quiver of $D^{b}(\operatorname{rep}(Q))$ containing projective representations. It is unique if and only if $Q$ is connected. The following result strengthens [13, Prop. 3.1.10].

Proposition 9.6. Any thick subcategory generated by a finite set of objects in connecting components of $D^{b}(\operatorname{rep}(Q))$ is generated by an exceptional sequence $E$ where all the terms can be chosen to be in connecting components.

Proof. It is sufficient to consider the case where $Q$ is connected and non-Dynkin. In [13, it is proven that if $\mathcal{C}$ is a thick subcategory of $\operatorname{rep}(Q)$ which is generated by a finite set of preprojective representations, then $\mathcal{C}$ is generated by an exceptional sequence where each term can be chosen to be preprojective. Let $\mathcal{A}$ be a thick subcategory of $D^{b}(\operatorname{rep}(Q))$ generated by objects $X_{1}, \ldots, X_{r}$ where all the $X_{i}$ lie in the connecting component and hence are exceptional. Let $\tau$ denote the Auslander-Reiten translate in $D^{b}(\operatorname{rep}(Q))$. The connecting component contains only the preprojective representations and the inverse shifts of the preinjective representations. There exists a positive integer $t$ for which all $\tau^{-t} X_{i}$ are preprojective indecomposable representations. Hence $\mathcal{C}\left(\tau^{-t} X_{1}, \ldots, \tau^{-t} X_{r}\right)$ is a thick subcategory of $\operatorname{rep}(Q)$ generated by preprojective representations. Therefore, by [13, Prop. 3.1.10],

$$
\mathcal{C}\left(\tau^{-t} X_{1}, \ldots, \tau^{-t} X_{r}\right)=\mathcal{C}(E)
$$

for an exceptional sequence $E=\left(Y_{1}, \ldots, Y_{m}\right)$ where all $Y_{i}$ are preprojective. Clearly, we also have

$$
\mathcal{D}\left(\tau^{-t} X_{1}, \ldots, \tau^{-t} X_{r}\right)=\mathcal{D}(E)
$$


where here, $E$ is seen as an exceptional sequence in $D^{b}(\operatorname{rep}(Q))$. From this, we see that

$$
\mathcal{A}=\mathcal{D}\left(X_{1}, \ldots, X_{r}\right)=\mathcal{D}\left(E^{\prime}\right)
$$

where $E^{\prime}$ is the exceptional sequence $E^{\prime}=\left(\tau^{t} Y_{1}, \ldots, \tau^{t} Y_{m}\right)$.

Using the fact that the thick subcategories $\mathcal{D}$ of $D^{b}(\operatorname{rep}(Q))$ correspond to the thick subcategories $H^{0}(\mathcal{D})$ of $\operatorname{rep}(Q)$, we get the following result.

Corollary 9.7. Let $Q$ be a connected acyclic quiver. Any thick subcategory of $\operatorname{rep}(Q)$ generated by non-regular representations is generated by an exceptional sequence whose terms can be chosen to be non-regular.

For the rest of this section, we specialize to the Euclidean case. Let $Q$ be a Euclidean quiver and let $E=\left(X_{1}, X_{2}, \ldots, X_{n}\right)$ be a full exceptional sequence in $\operatorname{rep}(Q)$. Let $s$ be an integer with $1 \leq s \leq n$. Clearly, one of the $X_{i}$ is not regular since $E$ is full. Then, by Lemma 4.3 one of $\mathcal{C}\left(E_{\leq s}\right), \mathcal{C}\left(E_{>s}\right)$ only contains indecomposable representations that are exceptional. Since each of $\mathcal{C}\left(E_{\leq s}\right), \mathcal{C}\left(E_{>s}\right)$ is equivalent to the category of representations of some acyclic quiver, we get that one of $\mathcal{C}\left(E_{\leq s}\right), \mathcal{C}\left(E_{>s}\right)$ is representation-finite. The following theorem also appears in [13, Theorem 3.2.15].

Theorem 9.8. (Dichev) Let $Q$ be a Euclidean quiver. Any thick subcategory of $\operatorname{rep}(Q)$ is either of the form $\mathcal{C}(E)$ for an exceptional sequence $E$ in $\operatorname{rep}(Q)$ or is entirely contained in $\mathcal{R}$ eg.

Proof. Let $\mathcal{A}$ be a thick subcategory of $\operatorname{rep}(Q)$ which contains at least one preprojective or preinjective indecomposable object $X$. By Proposition 9.1, $\mathcal{A}$ is a Hom-finite hereditary abelian category containing $X$. Clearly, $X$ is an exceptional representation, hence providing an exceptional sequence $E^{\prime}=(X)$ in $\mathcal{A}$. From Lemma 4.3, $\mathcal{D}\left(E^{\prime}\right)^{\perp}$ in $D^{b}(\mathcal{A})$ only contains exceptional objects. By Lemma 9.3. we see that $E^{\prime}$ can be completed to a full exceptional sequence $E$ in $D^{b}(\mathcal{A})$, proving that $\mathcal{A}$ is also generated by an exceptional sequence.

The following lemma is well known; see for example [19]. We will need it shortly.

Lemma 9.9. Let $R$ be a Dynkin quiver. Any thick subcategory of $\operatorname{rep}(R)$ is equivalent to $V^{\perp}$ for a rigid representation $V \in \operatorname{rep}(R)$. Moreover, $V^{\perp}$ is equivalent to $\operatorname{rep}\left(R^{\prime}\right)$, where $R^{\prime}$ is a (possibly disconnected) Dynkin quiver.

In order to understand thick subcategories of $\operatorname{rep}(Q)$ which are contained in $\mathcal{R}$ eg, we need to classify the thick subcategories of a single tube. Let $\mathcal{T}$ be a tube of rank $r$, which is identified with the additive subcategory of $\operatorname{rep}(Q)$ that it generates. Let $J$ be a subset of the quasi-simples of $\mathcal{T}$. Write $\mathcal{E}_{J}$ for $X^{\perp} \cap \mathcal{T}$, where $X$ is the direct sum of the quasi-simples not in $J$.

We say that $\left(\mathcal{E}_{J}, \mathcal{F}\right)$ is a regular orthogonal pair if $\mathcal{F} \subseteq{ }^{\perp} \mathcal{E}_{J} \cap \mathcal{E}_{J}^{\perp}, \mathcal{F}$ is thick in $\mathcal{T}$ and contains only exceptional indecomposables. In this case, let $\mathcal{S}_{J, \mathcal{F}}$ be the additive hull of $\mathcal{E}_{J}$ and $\mathcal{F}$, which is clearly a thick subcategory of $\mathcal{T}$. The following result describes all the thick subcategories in a given stable tube. It extends [13, Prop. 3.2.8], where connected thick subcategories are considered.

Proposition 9.10. Let $\mathcal{T}$ be a tube in $\operatorname{rep}(Q)$. Any thick subcategory of $\mathcal{T}$ can be written as $\mathcal{S}_{J, \mathcal{F}}$ for a unique subset $J$ of the quasi-simples and subcategory $\mathcal{F}$ such that $\left(\mathcal{E}_{J}, \mathcal{F}\right)$ is a regular orthogonal pair. 
Proof. Let $\mathcal{C}$ be a thick subcategory of $\mathcal{T}$. Let $J$ be the set of quasi-socles of the singular-isotropic representations in $\mathcal{C}$, if any. We claim that $\mathcal{E}_{J}$ is contained in $\mathcal{C}$, and that if we set $\mathcal{F}$ to be the additive hull of ind $\mathcal{C} \backslash$ ind $\mathcal{E}_{J}$, then $\left(\mathcal{E}_{J}, \mathcal{F}\right)$ is a regular orthogonal pair, so $\mathcal{C}=\mathcal{S}_{J, \mathcal{F}}$.

First, we establish that $\mathcal{E}_{J}$ is contained in $\mathcal{C}$. If $J=\emptyset$, then $\mathcal{E}_{J}=0$, so this is obvious. Assume otherwise. Let $X$ be the direct sum of the quasi-simples not in $J$. Then $\mathcal{E}_{J}=\mathcal{T} \cap X^{\perp}$. Suppose that $\mathcal{T}$ has rank $r_{1}$. Since the summands of $X$ form an exceptional sequence, from Proposition 4.2. $X^{\perp}$ is equivalent to the category of representations of a quiver $Q^{\prime}$ with $\left|Q_{0}\right|-r_{1}+|J|$ vertices. It is clear that $Q^{\prime}$ is a possibly disconnected Euclidean quiver. Since $\mathcal{T} \cap X^{\perp}$ contains exactly $|J|$ singular-isotropic representations, we see that the ranks of the tubes of $\operatorname{rep}\left(Q^{\prime}\right)$ will be the same as the ones for $\operatorname{rep}(Q)$, but one rank will decrease by $r_{1}-|J|$. If the $r_{i}$ denote the ranks of the non-homogeneous tubes for $\operatorname{rep}(Q)$, the well know formula $\sum\left(r_{i}-1\right)=n-2$ gives $(|J|-1)+\sum_{i \neq 1}\left(r_{i}-1\right)=\left(n-r_{1}+|J|\right)-2$ which then tells us that $Q^{\prime}$ is connected. It follows that $\mathcal{E}_{J}$ is equivalent to some tube $\mathcal{T}^{\prime}$ of $\operatorname{rep}\left(Q^{\prime}\right)$. Since the singular-isotropic representations in $\mathcal{T}^{\prime}$ generate all of $\mathcal{T}^{\prime}$ as a thick subcategory, it follows that the smallest thick subcategory containing the singular-isotropic representations in $\mathcal{C}$ is $\mathcal{E}_{J}$. Thus, $\mathcal{E}_{J}$ is contained in $\mathcal{C}$.

Suppose that $J \neq \emptyset$. Let $\mathcal{Q}$ denote the set of objects of $\mathcal{E}_{J}$ which correspond to the quasi-simples of $\mathcal{T}^{\prime}$. The objects in $\mathcal{E}_{J}$ consist of representations which have filtrations by objects from $\mathcal{Q}$.

Now think of the filtration by quasi-simples of the objects from $\mathcal{Q}$. Each $Q$ in $\mathcal{Q}$ has a filtration $\mathcal{K}_{Q}$ by a consecutive sequence of the quasi-simples; these consecutive sequences are disjoint and their union is the set of all the quasi-simples of $\mathcal{T}$. Suppose we have an indecomposable object $X \in \mathcal{F}$. Consider its filtration by quasi-simples, which also gives rise to a consecutive sequence of quasi-simples. Since $X \notin \mathcal{E}_{J}$, this sequence of quasi-simples is not the concatenation of subsequences corresponding to elements of $\mathcal{Q}$ : it either begins, or ends, or both, out of step with the subdivision of quasi-simples of $\mathcal{T}$ into the sets $\mathcal{K}_{Q}$. We would like to show that the quasi-simples in the filtration of $X$ all lie inside $\mathcal{K}_{Q}$ for some $Q$, and do not include either the quasi-socle or the quasi-top of that $Q$. Suppose that this is not the case. Then there is some $Q \in \mathcal{Q}$ such that $X$ admits a non-epimorphism to $Q$, or a non-monomorphism from $Q$. Suppose we are in the first case. (The second is dual.) Since $\mathcal{C}$ is thick, by Proposition $9.1 \mathcal{C}$ contains the image $A$ of $X$ in $Q$, so that there is a short exact sequence $0 \rightarrow A \rightarrow Z \rightarrow B \rightarrow 0$ in $\mathcal{C}$, where $Z$ is singular-isotropic and lies in $\mathcal{E}_{J}$. But then, there is another short exact sequence $0 \rightarrow B \rightarrow Z^{\prime} \rightarrow A \rightarrow 0$ where $Z^{\prime}$ is singular-isotropic. Since $\mathcal{C}$ is extension-closed, $Z^{\prime} \in \mathcal{C}$ and since $Z^{\prime}$ is singular-isotropic, we must have $Z^{\prime} \in \mathcal{E}_{J}$. This means that there exists $Q^{\prime} \in \mathcal{Q}$ which lies on the co-ray where $Z^{\prime}, X$, and $A$ lie. This contradicts the fact that $\mathcal{K}_{Q}$ and $\mathcal{K}_{Q^{\prime}}$ have to be disjoint. Therefore, we know that, for any indecomposable $X$ in $\mathcal{F}$, there is some $Q \in \mathcal{Q}$ such that $X$ admits a filtration by the quasi-simples in the filtration of $Q$, excluding its quasi-socle and quasi-top. This implies, in particular, that $X \subseteq{ }^{\perp} \mathcal{E}_{J} \cap \mathcal{E}_{J}^{\perp}$. It also shows that $X$ is necessarily exceptional.

Now we consider the case that $J=\emptyset$. The situation which we must rule out is that $\mathcal{C}$ contains some non-rigid indecomposables, but no singular-isotropic representations. That this is impossible is a by-product of the proof of Lemma 7.7. 
Now we are able to describe the semi-stable subcategories of $\operatorname{rep}(Q)$ which lie in Reg.

Proposition 9.11. A thick subcategory of $\mathcal{R} e g$ is semi-stable if and only if it contains all the homogeneous tubes and its intersection with each non-homogeneous tube $\mathcal{T}_{i}$ is of the form $\mathcal{S}_{J_{i}, \mathcal{F}_{i}}$ where each $J_{i}$ is non-empty.

Proof. By Theorem 5.5, a semi-stable subcategory of $\mathcal{R}$ eg can also be written as $\mathcal{A} \cap$ $\mathcal{R}$ eg for $\mathcal{A}$ some finitely generated extension-closed abelian subcategory, equivalent to the representations of a (possibly disconnected) Euclidean quiver. Since $\mathcal{A}$ is finitely generated, it can be written as $V^{\perp}$ for some rigid object $V$, and since $\mathcal{A}$ is equivalent to a possibly disconnected Euclidean quiver, $V$ must be regular.

Since $V$ is regular, $V^{\perp}$ contains all the homogeneous tubes. Now consider $V_{i}$, the maximal direct summand of $V$ lying in $\mathcal{T}_{i}$. Since $V_{i}$ is rigid, $V_{i}$ is contained in some wing, and thus $V_{i}^{\perp}$ contains some singular-isotropic representation. It follows that $V^{\perp} \cap \mathcal{T}_{i}$ is of the form $\mathcal{S}_{J_{i}, \mathcal{F}_{i}}$ where $J_{i}$ is non-empty.

Conversely, suppose we have a thick subcategory as described in the statement of the proposition. We want to show that it is of the form $V^{\perp} \cap \mathcal{R}$ eg where $V$ is rigid. Clearly, it suffices to consider the case of one tube $\mathcal{T}$, and a subcatgory $\mathcal{S}_{J, \mathcal{F}}$, with $J \neq \emptyset$. We want to show that there is some rigid representation $V \in \mathcal{T}$ such that $V^{\perp} \cap \mathcal{T}=\mathcal{S}_{J, F}$.

Let $\mathcal{Q}$ be the collection of the (relative) quasi-simples of $\mathcal{E}_{J}$, as before. For each $A \in \mathcal{Q}$, let $R_{A}$ be the target of the irreducible epimorphism from $A$ (or $R_{A}=0$ if there is no such epimorphism). For $A, B \in \mathcal{Q}$, if $\operatorname{Ext}\left(R_{A}, R_{B}\right) \neq 0$, then there is a nonzero morphism $g: R_{B} \rightarrow \tau R_{A}$. Since there is an epimorphism $f: B \rightarrow R_{B}$ and a monomorphism $h: \tau R_{A} \rightarrow A$, this yields a nonzero radical morphism $h g f: B \rightarrow$ $A$, contradicting that $A, B$ are quasi-simples. Therefore, $\bigoplus_{A \in \mathcal{Q}} R_{A}$ is rigid. For $A \in \mathcal{Q}$, denote by $\mathcal{W}_{A}$ the extension-closed abelian subcategory generated by all the quasi-simple composition factors of $A$ except its quasi-top and quasi-socle. We see that $\left(\bigoplus_{A \in \mathcal{Q}} R_{A}\right)^{\perp}$ consists of the additive hull of $\mathcal{E}_{J}$ together with each of the wings $\mathcal{W}_{A}$. Since each of the $\mathcal{W}_{A}$ is representation-finite of type $\mathbb{A}$, Lemma 9.9 tells us that if we add further summands to $\bigoplus R_{A}$, it is possible to find a rigid object $V$ such that $V^{\perp} \cap \mathcal{T}=\mathcal{S}_{J, \mathcal{F}}$.

Using the classification of thick subcategories inside the regular representations, the previous proposition can be restated as follows:

Proposition 9.12. The semi-stable subcategories in (ii) of Theorem 5.5 can also be described as those abelian, extension-closed subcategories of the regular part of $\operatorname{rep}(Q)$, which contain infinitely many indecomposable objects from each tube.

We end this section with the following, which will be used in the next section.

Proposition 9.13. Let $Q$ be a Euclidean quiver and $\mathcal{C}$ be a thick subcategory of $\operatorname{rep}(Q)$. Then one of $\mathcal{C}, \mathcal{C}^{\perp}$ is not contained in $\mathcal{R}$ eg if and only if both $\mathcal{C}$ and $\mathcal{C}^{\perp}$ are generated by exceptional sequences. In this case, $\left(\mathcal{C}, \mathcal{C}^{\perp}\right)$ is an exceptional semi-orthogonal pair.

Proof. If $\mathcal{C}$ is not contained in $\mathcal{R}$ eg, then it follows from Theorem 9.8 that $\mathcal{C}$ is generated by an exceptional sequence. Since this exceptional sequence can be completed to a full exceptional sequence, see 9 , $\mathcal{C}^{\perp}$ is also generated by an exceptional sequence. Similarly, if $\mathcal{C}^{\perp}$ is not contained in $\mathcal{R}$ eg, then $\mathcal{C}^{\perp}$ is generated by an exceptional sequence by Theorem 9.8. By Lemma 9.4 we have ${ }^{\perp}\left(\mathcal{C}^{\perp}\right)=\mathcal{C}$ and hence $\mathcal{C}$ is 
generated by an exceptional sequence. Suppose now that both $\mathcal{C}, \mathcal{C}^{\perp}$ are contained in $\mathcal{R}$ eg. If $\mathcal{C}$ is generated by an exceptional sequence, then all the representations in homogeneous tubes are contained in $\mathcal{C}^{\perp}$ and hence, $\mathcal{C}^{\perp}$ cannot be generated by an exceptional sequence. If $\mathcal{C}^{\perp}$ is generated by an exceptional sequence, then all the representations in homogeneous tubes are contained in ${ }^{\perp}\left(\mathcal{C}^{\perp}\right)=\mathcal{C}$ and $\mathcal{C}$ cannot be generated by an exceptional sequence.

\section{Intersection of SEMi-STABLE SUbCATEGORIES}

In this section, we start with $Q$ any connected acyclic quiver and later specialize to the Euclidean case. We first look at some situations where the intersection of semi-stable subcategories is again semi-stable, and we end the section by describing, in the Euclidean case, how to construct the whole set of subcategories of $\operatorname{rep}(Q)$ arising as an intersection of semi-stable subcategories. As already seen, we may assume that our semi-stable subcategories are of the form $\operatorname{rep}(Q)_{d}$ where $d$ is a dimension vector.

A prehomogeneous dimension vector $d$ such that all the indecomposable summands of $M(d)$ are non-regular will be called strongly prehomogeneous. The following result says that when $d_{1}, d_{2}$ are strongly prehomogeneous, the intersection $\operatorname{rep}(Q)_{d_{1}} \cap \operatorname{rep}(Q)_{d_{2}}$ remains semi-stable.

Proposition 10.1. Let $d_{1}, d_{2}$ be two strongly prehomogeneous dimension vectors. Then $\operatorname{rep}(Q)_{d_{1}} \cap \operatorname{rep}(Q)_{d_{2}}=\operatorname{rep}(Q)_{d_{3}}$, where $d_{3}$ is prehomogeneous.

Proof. By assumption, $\operatorname{rep}(Q)_{d_{i}}=M\left(d_{i}\right)^{\perp}, i=1,2$, where the $M\left(d_{i}\right)$ are rigid representations whose indecomposable direct summands are non-regular. Now, $\operatorname{rep}(Q)_{d_{1}} \cap \operatorname{rep}(Q)_{d_{2}}=\mathcal{C}\left(M\left(d_{1}\right), M\left(d_{2}\right)\right)^{\perp}$. However, from Corollary 9.7 .

$$
\mathcal{C}\left(M\left(d_{1}\right), M\left(d_{2}\right)\right)=\mathcal{C}(E),
$$

where $E$ is an exceptional sequence whose terms are non-regular. This gives a rigid representation $V$ with $\mathcal{C}\left(M\left(d_{1}\right), M\left(d_{2}\right)\right)=\mathcal{C}(V)$ by Proposition 4.4 Then, $\operatorname{rep}(Q)_{d_{1}} \cap \operatorname{rep}(Q)_{d_{2}}=V^{\perp}$. Since $V$ is rigid, the orbit of $V$ is open in $\operatorname{rep}\left(Q, d_{V}\right)$. Hence, the canonical decomposition of $V$ is given by the dimension vectors of its indecomposable direct summands. In particular, $d_{3}$ is prehomogeneous. 10.1 .

Here is a simple examples that illustrate some results of Section 9 and Proposition

Example 10.2. Let $Q$ be the following quiver

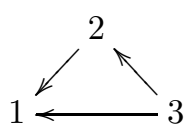

There is only one non-homogeneous tube in $\operatorname{rep}(Q)$. It is of rank two with two quasi-simple representations $M_{1}, M_{2}$ where $d_{M_{1}}=(1,0,1)$ and $d_{M_{2}}=(0,1,0)$. Observe that $\mathcal{C}\left(M_{1}, M_{2}\right)$ is not generated by an exceptional sequence. This shows that the assumption that $M_{1}, M_{2}$ are non-regular in Corollary 9.7 is essential.

For $i=1,2,3$, denote by $S_{i}$ the simple representation at $i$, by $P_{i}$ the projective representation at $i$ and by $I_{i}$ the injective representation at $i$. Observe that $d_{S_{1}}, d_{S_{3}}$ are strongly prehomogeneous. Consider $\operatorname{rep}(Q)_{d_{S_{1}}} \cap \operatorname{rep}(Q)_{d_{S_{3}}}=\operatorname{add}\left(I_{2}\right)$. By Proposition 10.1 we can write $\operatorname{rep}(Q)_{d_{S_{1}}} \cap \operatorname{rep}(Q)_{d_{S_{3}}}$ as $\operatorname{rep}(Q)_{d_{3}}$ for some $d_{3}$ which 
is prehomogeneous but not necessarily strongly prehomogeneous. We will now determine $d_{3}$.

Observe that $\operatorname{rep}(Q)_{d_{3}}=\operatorname{add}\left(I_{2}\right)=V^{\perp}$ for some $V$. Since $I_{2}$ is supported at vertices 2,3 , we have that $\left.\left\langle d_{V},-\right\rangle\right|_{Q^{\prime}}=\left.\left\langle d_{3},-\right\rangle\right|_{Q^{\prime}}$ where $Q^{\prime}$ is the full subquiver of $Q$ generated by vertices 2,3 ; see Corollary 3.2, This gives $\left.d_{V}\right|_{Q^{\prime}}=\left.d_{3}\right|_{Q^{\prime}}$. The condition $\left\langle d_{V}, d_{I_{2}}\right\rangle=0$ gives $d_{V}=(a, 0, b)$ for some $a, b \geq 0$. Hence, $d_{3}=(c, 0, b)$ for some integer $c$. This gives that $I_{2}$ lies in $\operatorname{rep}(Q)_{(c, 0, b)}$ for any $c, b$ with $b \geq 0$. However, for some values of $b, c, \operatorname{rep}(Q)_{(c, 0, b)}$ properly contains $\operatorname{add}\left(I_{2}\right)$. If $c=b$, then by Proposition 5.1, $S_{1}$ lies in $\operatorname{rep}(Q)_{(c, 0, b)}$, which is impossible. Similarly, if $c=0$, then $M_{1}$ lies in $\operatorname{rep}(Q)_{(c, 0, b)}$, which is impossible. Finally, if $b=0$, then $S_{2}$ lies in $\operatorname{rep}(Q)_{(c, 0, b)}$, which is again impossible. Hence, $b, c$ are non-zero and are not equal. An easy check gives that $d_{3}=(c, 0, b)$ is cp-equivalent to one of the vectors $(1,0,2),(2,0,1),(-1,0,1)$ whose canonical presentations are $(1,0,1)+$ $(0,0,1),(1,0,1)+(1,0,0),(-1,0,0)+(0,0,1)$, respectively.

Let $d_{1}=(1,0,0)$ and $d_{2}=(2,1,1)$. Since $d_{1}$ is the dimension vector of $P_{1}$, we have

Similarly,

$$
\operatorname{rep}(Q)_{d_{1}}=P_{1}^{\perp}=\operatorname{add}\left(S_{2}, S_{3}, I_{2}\right)=\mathcal{C}\left(I_{3}, I_{2}\right) .
$$

$$
\operatorname{rep}(Q)_{d_{2}}=P_{3}^{\perp}=\operatorname{add}\left(S_{1}, S_{2}, P_{2}\right)=\mathcal{C}\left(P_{1}, P_{2}\right) .
$$

Therefore, each of $\operatorname{rep}(Q)_{d_{1}}, \operatorname{rep}(Q)_{d_{2}}$ is generated by a representation whose indecomposable direct summands are non-regular. We see that the intersection $\operatorname{rep}(Q)_{d_{1}} \cap \operatorname{rep}(Q)_{d_{2}}$ is $\mathcal{C}\left(P_{1}, P_{3}\right)^{\perp}=\operatorname{add}\left(S_{2}\right)$, where $S_{2}$ is regular.

From now on, we suppose that $Q$ is a Euclidean quiver. We give a complete description of the possible intersections of semi-stable subcategories of $\operatorname{rep}(Q)$, and in particular, a description of those intersections that are not semi-stable. Let us start with some notations and reminders.

Given two semi-orthogonal pairs $\left(\mathcal{A}, \mathcal{A}^{\perp}\right)$ and $\left(\mathcal{B}, \mathcal{B}^{\perp}\right)$, we define

$$
\left(\mathcal{A}, \mathcal{A}^{\perp}\right) *\left(\mathcal{B}, \mathcal{B}^{\perp}\right)=\left(\mathcal{C}(\mathcal{A}, \mathcal{B}), \mathcal{A}^{\perp} \cap \mathcal{B}^{\perp}\right)
$$

where we recall that $\mathcal{C}(\mathcal{A}, \mathcal{B})$ denotes the smallest thick subcategory of $\operatorname{rep}(Q)$ containing $\mathcal{A}$ and $\mathcal{B}$. Observe that $\mathcal{A}^{\perp} \cap \mathcal{B}^{\perp}=\mathcal{C}(\mathcal{A}, \mathcal{B})^{\perp}$, hence defining a new semi-orthogonal pair $\left(\mathcal{C}(\mathcal{A}, \mathcal{B}), \mathcal{A}^{\perp} \cap \mathcal{B}^{\perp}\right)$.

Recall that for each facet $F_{I}$ of $H_{\delta}^{s s}$, we have the representation-finite thick subcategory $\mathcal{W}_{I}$ generated by the exceptional representations whose dimension vectors lie in $F_{I}$. We also have the simplicial cone $C_{I}$ which is generated by $F_{I}$ and $\delta$. Given a dimension vector $d$, recall that $M(\hat{d})$ is a rigid representation of dimension vector $\hat{d}$, where $\hat{d}$ is the sum of the real Schur roots appearing in the canonical decomposition of $d$. We start with the following.

Proposition 10.3. Let $Q$ be a Euclidean quiver and $d_{1}, d_{2}$ be dimension vectors. Then $\left(\mathcal{C}\left(M\left(\hat{d}_{1}\right)\right), \mathcal{C}\left(M\left(\hat{d}_{1}\right)\right)^{\perp}\right) *\left(\mathcal{C}\left(M\left(\hat{d}_{2}\right)\right), \mathcal{C}\left(M\left(\hat{d}_{2}\right)\right)^{\perp}\right)$ is exceptional if and only if one of the following occurs:

(a) At least one of $d_{1}, d_{2}$ does not lie on $H_{\delta}^{s s}$,

(b) There exists $I \in R$ with $d_{1}, d_{2} \in C_{I}$.

Proof. If at least one of $d_{1}, d_{2}$ is not in $H_{\delta}^{s s}$, then $\mathcal{C}\left(M\left(\hat{d}_{1}\right), M\left(\hat{d}_{2}\right)\right)$ is not contained in $\mathcal{R}$ eg. We apply Proposition 9.13 in this case. If both $d_{1}, d_{2}$ lie in the same $C_{I}$, then $\mathcal{C}\left(M\left(\hat{d}_{1}\right), M\left(\hat{d}_{2}\right)\right)$ is clearly representation-finite by Lemma 8.1, By 
Lemma 9.5, $\mathcal{C}\left(M\left(\hat{d}_{1}\right), M\left(\hat{d}_{2}\right)\right)$ is generated by an exceptional sequence and so is $\mathcal{C}\left(M\left(\hat{d}_{1}\right), M\left(\hat{d}_{2}\right)\right)^{\perp}$. Suppose now that $d_{1}, d_{2}$ both lie in $H_{\delta}^{s s}$ but in different cones. If $\mathcal{C}\left(M\left(\hat{d}_{1}\right), M\left(\hat{d}_{2}\right)\right)$ is representation-finite, then it lies in some $\mathcal{W}_{I}$ by Lemma 8.1 and hence both $d_{1}, d_{2}$ lie in $C_{I}$, a contradiction. Since $\mathcal{C}\left(M\left(\hat{d}_{1}\right), M\left(\hat{d}_{2}\right)\right)^{\perp}$ contains all the representations in homogeneous tubes, it is not representation-finite. Therefore, $\left(\mathcal{C}\left(M\left(\hat{d}_{1}\right)\right), \mathcal{C}\left(M\left(\hat{d}_{1}\right)\right)^{\perp}\right) *\left(\mathcal{C}\left(M\left(\hat{d}_{2}\right)\right), \mathcal{C}\left(M\left(\hat{d}_{2}\right)\right)^{\perp}\right)$ cannot be exceptional since neither $\mathcal{C}\left(\mathcal{C}\left(M\left(\hat{d}_{1}\right)\right), \mathcal{C}\left(M\left(\hat{d}_{2}\right)\right)\right)$ nor $\mathcal{C}\left(\mathcal{C}\left(M\left(\hat{d}_{1}\right)\right), \mathcal{C}\left(M\left(\hat{d}_{2}\right)\right)\right)^{\perp}$ is representation-finite.

From what we just proved, if $\left(\mathcal{C}\left(M\left(\hat{d}_{1}\right)\right), \mathcal{C}\left(M\left(\hat{d}_{1}\right)\right)^{\perp}\right) *\left(\mathcal{C}\left(M\left(\hat{d}_{2}\right)\right), \mathcal{C}\left(M\left(\hat{d}_{2}\right)\right)^{\perp}\right)$ is not exceptional, then both $\mathcal{C}\left(M\left(\hat{d}_{1}\right), M\left(\hat{d}_{2}\right)\right)$ and $\mathcal{C}\left(M\left(\hat{d}_{1}\right)\right)^{\perp} \cap \mathcal{C}\left(M\left(\hat{d}_{2}\right)\right)^{\perp}$ are contained in $\mathcal{R}$ eg and are not representation-finite. The following proposition gives a first partial answer on how to compute the intersection of two semi-stable subcategories in the Euclidean case.

Proposition 10.4. Let $Q$ be a Euclidean quiver and $d_{1}, d_{2}$ be dimension vectors satisfying the equivalent conditions of Proposition 10.3. Then $\operatorname{rep}(Q)_{d_{1}} \cap \operatorname{rep}(Q)_{d_{2}}=$ $\operatorname{rep}(Q)_{d_{3}}$ for some dimension vector $d_{3}$.

(a) If at least one of $d_{1}, d_{2}$ is not in $H_{\delta}^{s s}$, then $d_{3}$ is not in $H_{\delta}^{s s}$. In particular, $\operatorname{rep}(Q)_{d_{3}}$ is representation-finite.

(b) If both $d_{1}, d_{2}$ lie on some facet $F_{I}$, then $d_{3}$ can be chosen to be in $F_{I}$ and $\operatorname{rep}(Q)_{d_{3}}=V^{\perp}$ where $V$ is rigid and $\mathcal{C}(V)$ is representation-finite.

(c) If both $d_{1}, d_{2}$ lie in some $C_{I}$ but not both on $F_{I}$, then $d_{3}$ can be chosen to be in $C_{I}$. In this case, $\operatorname{rep}(Q)_{d_{3}}=V^{\perp} \cap \mathcal{R}$ eg where $V$ is rigid and $\mathcal{C}(V)$ is representation-finite.

Proof. The main statement follows from Propositions $4.4,5.4$ and 10.3 , For $(a)$, suppose that $d_{1}$ does not lie in $H_{\delta}^{s s}$. Then $\operatorname{rep}(Q)_{d_{1}}$ is representation-finite and so is $\operatorname{rep}(Q)_{d_{1}} \cap \operatorname{rep}(Q)_{d_{2}}=\operatorname{rep}(Q)_{d_{3}}$. If $d_{3}$ lies in $H_{\delta}^{s s}$, then all the homogeneous tubes are contained in $\operatorname{rep}(Q)_{d_{3}}$, contradicting that $\operatorname{rep}(Q)_{d_{3}}$ is representationfinite. For $(b), d_{1}=\hat{d}_{1}$ and $d_{2}=\hat{d}_{2}$ and we have $M\left(d_{1}\right), M\left(d_{2}\right) \in \mathcal{W}_{I}$, and hence, $\mathcal{C}\left(M\left(d_{1}\right), M\left(d_{2}\right)\right) \subseteq \mathcal{W}_{I}$ is representation-finite. Therefore, $\mathcal{C}\left(M\left(d_{1}\right), M\left(d_{2}\right)\right)$ is generated by some rigid representation $V$ and $\operatorname{rep}(Q)_{d_{3}}=V^{\perp}$. For part $(c)$, observe that, for $i=1,2$, we have $\operatorname{rep}(Q)_{d_{i}}=\operatorname{rep}(Q)_{\hat{d}_{i}} \cap \mathcal{R}$ eg where $\hat{d}_{i} \in F_{I}$. Hence, from part $(b)$, the intersection $\operatorname{rep}(Q)_{d_{1}} \cap \operatorname{rep}(Q)_{d_{2}}$ is given by $\operatorname{rep}(Q)_{d_{3}^{\prime}} \cap \mathcal{R}$ eg where $d_{3}^{\prime} \in F_{I}$ and $\operatorname{rep}(Q)_{d_{3}^{\prime}}=V^{\perp}$ for some rigid $V$ such that $\mathcal{C}(V)$ is representation-finite. The result follows by setting $d_{3}=d_{3}^{\prime}+\delta$.

Now, we concentrate on the remaining case, that is, to describe the intersection $\operatorname{rep}(Q)_{d_{1}} \cap \operatorname{rep}(Q)_{d_{2}}$ when both $d_{1}$ and $d_{2}$ lie in $H_{\delta}^{s s}$, and not in the same $C_{I}$. We can clearly assume that $Q$ has more than 2 vertices.

Lemma 10.5. If $d_{1}$ and $d_{2}$ both lie in $H_{\delta}^{s s}$, and do not both lie in the same $C_{I}$, then $\operatorname{rep}(Q)_{d_{1}} \cap \operatorname{rep}(Q)_{d_{2}}$ is contained in $\mathcal{R}$ eg.

Proof. We can clearly assume, from Proposition 5.4 that both $d_{1}, d_{2}$ lie on the boundary of $H_{\delta}^{s s}$. Let $\mathcal{C}=\operatorname{rep}(Q)_{d_{1}} \cap \operatorname{rep}(Q)_{d_{2}}$. Let $f=d_{1}+d_{2}$. We observe that $X=M\left(d_{1}\right) \oplus M\left(d_{2}\right)$ is a representation with dimension vector $f$, such that $\operatorname{Hom}(X, Y)=0$ and $\operatorname{Ext}^{1}(X, Y)=0$ for any $Y$ in $\mathcal{C}$. Thus $\mathcal{C}$ is contained in $\operatorname{rep}(Q)_{f}$. Since $d_{1}$ and $d_{2}$ do not both lie in the same $C_{I}$, we know that $f$ lies in the 
interior of $H_{\delta}^{s s}$. Proposition 5.4 tells us that $\operatorname{rep}(Q)_{f}$ is contained in $\mathcal{R}$ eg, proving the result.

Since we now know that, in the case we are presently studying, $\operatorname{rep}(Q)_{d_{1}} \cap$ $\operatorname{rep}(Q)_{d_{2}}$ is contained in $\mathcal{R}$ eg, it suffices to restrict our attention to the regular parts of $\operatorname{rep}(Q)_{d_{1}}$ and $\operatorname{rep}(Q)_{d_{2}}$. It therefore suffices to assume that neither $d_{i}$ lies on the boundary of $H_{\delta}^{s s}$. If $d_{1}$ does lie on the boundary, replace it by $d_{1}^{\prime}=d_{1}+\delta$, and observe that $\operatorname{rep}(Q)_{d_{1}^{\prime}}=\mathcal{R} \operatorname{eg} \cap \operatorname{rep}(Q)_{d_{1}}$.

By Proposition 9.11, we know exactly what kind of subcategories can arise as semi-stable subcategories of $\mathcal{R}$ eg: namely, they are the subcategories with the property that their intersection with each tube is of the form $\mathcal{S}_{J, \mathcal{F}}$ where $J$ is non-empty. Observe that the condition $J \neq \emptyset$ applied to a homogeneous tube $\mathcal{T}$ just means $\mathcal{S}_{J, \mathcal{F}}=\mathcal{T}($ and $\mathcal{F}=0)$.

Given a non-homogeneous tube $\mathcal{T}$ of $\operatorname{rep}(Q)$, denote by $\mathcal{O}_{\mathcal{T}}$ the set of indecomposable representations in $\mathcal{T}$ which admit an irreducible monomorphism to a singular-isotropic representation. The set $\mathcal{O}_{\mathcal{T}}$ hence form a $\tau$-orbit of $\mathcal{T}$, which lies just below the $\tau$-orbit of singular-isotropic representations.

Lemma 10.6. Let $\mathcal{T}$ be a non-homogeneous tube, and $\mathcal{S}_{J_{i}, \mathcal{F}_{i}}$ be thick subcategories of $\mathcal{T}$ with each $J_{i}$ non-empty. Let $\mathcal{C}=\bigcap_{i} \mathcal{S}_{J_{i}, \mathcal{F}_{i}}$. Then

(a) The category $\mathcal{C}$ is given by some $\mathcal{S}_{K, \mathcal{G}}$, where $\mathcal{G}$ does not contain any indecomposables from $\mathcal{O}_{\mathcal{T}}$.

(b) Conversely, any thick subcategory $\mathcal{S}_{K, \mathcal{G}}$ of $\mathcal{T}$ such that $\mathcal{G}$ does not contain any indecomposables from $\mathcal{O}_{\mathcal{T}}$ can be written as an intersection $\mathcal{S}_{K_{1}, \mathcal{G}_{1}} \cap \mathcal{S}_{K_{2}, \mathcal{G}_{2}}$ where $K_{1}, K_{2}$ are non-empty.

Proof. We begin by proving $(a)$. Clearly, $K=\bigcap_{i} J_{i}$. If $K \neq \emptyset$, let $V$ be a singular-isotropic representation contained in $\mathcal{C}$. Since $\mathcal{G} \subseteq{ }^{\perp} V \cap V^{\perp}$, it follows that $\mathcal{G} \cap \mathcal{O}_{\mathcal{T}}=\emptyset$, as desired.

Suppose now that $K=\emptyset$. Since $\bigcap_{i} \mathcal{E}_{J_{i}}=0$, any indecomposable of $\bigcap_{i} \mathcal{S}_{J_{i}, \mathcal{F}_{i}}$ must be contained in some $\mathcal{F}_{i}$. But by the previous argument, $\mathcal{F}_{i} \cap \mathcal{O}_{\mathcal{T}}=\emptyset$.

Now we prove the converse direction. We may assume that $K=\emptyset$, otherwise, we may take all $\mathcal{S}_{K_{i}, \mathcal{G}_{i}}$ equal $\mathcal{S}_{K, \mathcal{G}}$. Let $Z$ be a singular-isotropic representation in $\mathcal{T}$ with quasi-socle $S$. We claim that for an exceptional object $M$ in $\mathcal{T}$, we have $\operatorname{Hom}(M, Z) \neq 0$ if and only if $M$ has $S$ as a quasi-simple composition factor. Fix $M$ exceptional in $\mathcal{T}$. Suppose that $M$ has $S$ as a quasi-simple composition factor. If $M$ has $S$ as quasi-socle, then we have a monomorphism $M \rightarrow Z$ and $\operatorname{Hom}(M, Z) \neq 0$. Otherwise $M$ has a quotient $N$ that has $S$ as quasi-socle. The projection $M \rightarrow N$ followed by the monomorphism $N \rightarrow Z$ gives a nonzero morphism $M \rightarrow Z$ and hence, $\operatorname{Hom}(M, Z) \neq 0$. Conversely, assume that $M$ does not have $S$ as a quasisimple composition factor. Let $f: M \rightarrow Z$ be a morphism with image $C$. Since $S$ is the quasi-socle of $Z$, either $C=0$ or $S$ is the quasi-socle of $C$. The latter case gives that $S$ is a quasi-simple composition factor of $M$, a contradiction. Therefore, $C=0$ and hence $f=0$, which gives $\operatorname{Hom}(M, Z)=0$. This proves the claim. Similarly, one can prove that for an exceptional object $M$ in $\mathcal{T}$, we have $\operatorname{Ext}^{1}(M, Z) \neq 0$ if and only if $M$ has $S$ as a quasi-simple composition factor.

For an exceptional object $L$ in $\mathcal{O}_{T}$, denote by $\mathcal{W}_{L}$ the extension-closed abelian subcategory of $\mathcal{T}$ generated by the quasi-simple composition factors of $L$. Now from Lemma 8.1, we see that $\mathcal{G}$ is contained in $\mathcal{W}_{L}$ for some $L \in \mathcal{O}_{T}$. Observe that there is at least one quasi-simple object $S$ in $\mathcal{W}_{L}$ such that $S$ is not a quasi-simple 
composition factor of any object in $\mathcal{G}$. Otherwise, since $\mathcal{G}$ is thick, we would get $L \in \mathcal{G}$, a contradiction. Let $M_{1}$ be the indecomposable object of maximal quasilength in $\mathcal{W}_{L}$ that has $S$ as quasi-socle, and take $N_{1}=\tau^{-1} M_{1}$. Moreover, let $N_{2}$ be the indecomposable object of maximal quasi-length in $\mathcal{W}_{L}$ that has $S$ as quasi-top. Observe that $\mathcal{G} \subseteq\left(N_{1} \oplus N_{2}\right)^{\perp} \subseteq \mathcal{W}_{L}$. Now, $\left(N_{1} \oplus N_{2}\right)^{\perp}=\mathcal{C}_{1} \amalg \mathcal{C}_{2}$ where each $\mathcal{C}_{i}$, provided it is non-zero, is equivalent to the category of representations of a Dynkin quiver. From Lemma 9.9, there exists a rigid $C_{i} \in \mathcal{C}_{i}$ such that $\mathcal{G} \cap \mathcal{C}_{i}=C_{i}^{\perp} \cap \mathcal{C}_{i}$. Thus, we have $\mathcal{G}=\left(N_{1} \oplus N_{2} \oplus C_{1} \oplus C_{2}\right)^{\perp}$. By Proposition 9.10, each $\left(N_{i} \oplus C_{i}\right)^{\perp}$ can be expressed as some $\mathcal{S}_{K_{i}, \mathcal{G}_{i}}$, so $\mathcal{G}=\mathcal{S}_{K_{1}, \mathcal{G}_{1}} \cap \mathcal{S}_{K_{2}, \mathcal{G}_{2}}$. Now, $S$ is not a quasi-simple composition factor of $N_{1} \oplus C_{1}$, so $K_{1}$ contains $S$ and if $U$ is the unique quasi-simple object not in $\mathcal{W}_{L}$, then $U$ is not a quasi-simple composition factor of $N_{2} \oplus C_{2}$, so $U \in K_{2}$. It follows that the expression we have obtained for $\mathcal{G}$ as the intersection of $\mathcal{S}_{K_{1}, \mathcal{G}_{1}}$ and $\mathcal{S}_{K_{2}, \mathcal{G}_{2}}$ is of the desired form.

The following example illustrates the second part of the Lemma and the idea of its proof.

Example 10.7. In the picture below, we have a tube of rank 6 where only the exceptional indecomposable objects are drawn.

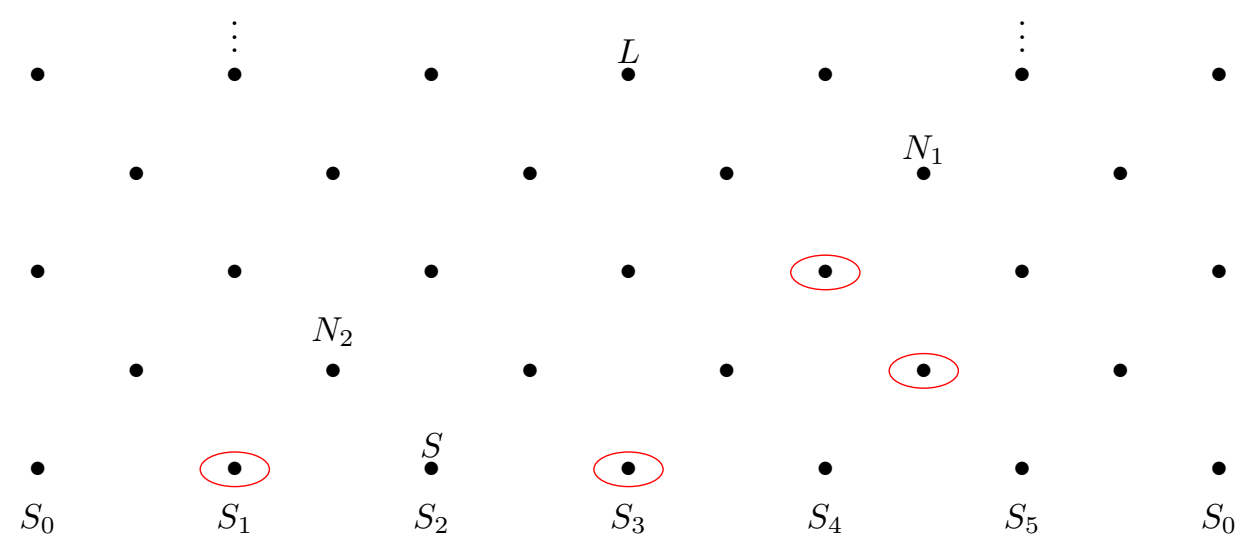

Figure 2. A tube of rank 6

We have a thick subcategory $\mathcal{G}$ whose indecomposable objects are circled. Using the notations of the proof of Lemma 10.6, take $C_{1}=S_{5}$ and $C_{2}=0$. Then each $\left(N_{i} \oplus C_{i}\right)^{\perp}$ is given by $S_{K_{i}, \mathcal{G}_{i}}$, where $K_{i}$ is non-empty.

We have now essentially proved our final main theorem.

Theorem 10.8. Let $Q$ be a Euclidean quiver. There are finitely many subcategories of $\operatorname{rep}(Q)$ which arise as an intersection of semi-stable subcategories, and which are not themselves semi-stable subcategories for any stability condition. Moreover, such a subcategory is characterized by the following.

(a) It is contained entirely in the regular part of $Q$.

(b) It contains all the homogeneous tubes.

(c) Its intersection with each non-homogeneous tube $\mathcal{T}_{i}$ can be written as $\mathcal{S}_{J_{i}, \mathcal{F}_{i}}$ where $\left(\mathcal{E}_{J_{i}}, \mathcal{F}_{i}\right)$ is a regular orthogonal pair in $\mathcal{T}_{i}$ with $\mathcal{F}_{i} \cap \mathcal{O}_{\mathcal{T}_{i}}=\emptyset$. Moreover, at least one of the $J_{i}$ is empty. 
(d) It can be expressed as the intersection of at most two semi-stable subcategories.

Proof. We have shown that if $\mathcal{C}_{1}$ and $\mathcal{C}_{2}$ are two semi-stable subcategories, such that $\mathcal{C}_{1} \cap \mathcal{C}_{2}$ is not itself semi-stable, then $\mathcal{C}_{1} \cap \mathcal{C}_{2}$ is contained in the regular part $\mathcal{R}$ eg, and contains the homogeneous tubes. Since it is thick, its intersection with each non-homogeneous tube can be written as $\mathcal{S}_{J_{i}, \mathcal{F}_{i}}$, and since, by assumption, it is not semi-stable, some $J_{i}$ must be empty. Lemma 10.6 says further that $\mathcal{F}_{i} \cap \mathcal{O}_{\mathcal{T}_{i}}=\emptyset$. The same lemma also shows that any such subcategory can be written as the intersection of at most two semi-stable subcategories.

ACKNOWLEDGMENTS: The authors are supported by NSERC while the second author is also supported in part by AARMS. The third author would like to thank MSRI for its hospitality during the completion of this paper. The authors would like to express their gratitude to an anonymous referee for a careful reading of the paper, which led to several improvements.

\section{REFERENCES}

[1] I. Assem, D. Simson and A. Skowroński, Elements of representation theory of associative algebras. Vol. 1., Techniques of representation theory. London Mathematical Society Student Texts 65, Cambridge University Press, Cambridge, 2006.

[2] A. Barvinok, A course in convexity, Graduate Studies in Mathematics 54, American Mathematical Society, Providence, RI, 2002.

[3] D. Bessis, The dual braid monoid, Ann Sci. École Norm. Sup. (4) 36 (2003), no. 5, 647-683.

[4] T. Brady, A partial order on the symmetric group and new $K(\pi, 1)$ 's for the braid groups. Adv. Math 161 (2001), no. 1, 20-40.

[5] T. Brady and C. Watt, $K(\pi, 1)$ 's for Artin groups of finite type. Proceedings of the Conference on Geometric and Combinatorial Group Theory,Part I (Haifa, 2000). Geom. Dedicata 94 (2002), 225-250.

[6] D. Chan, Splitting Bundles over Hereditary Orders, Comm. in Alg. 333 (7) (2005) 2193-9.

[7] D. Chan and C. Ingalls. Noncommutative Coordinate Rings and Stacks, Proc. of the LMS (3) 88 (2004) 63-88.

[8] C. Chindris, Cluster fans, stability conditions, and domains of semi-invariants, Trans. Amer. Math. Soc. 363 (2011), no. 4, 2171-2190.

[9] W. Crawley-Boevey, Exceptional sequences of representations of quivers, Proceedings of the Sixth International Conference on Representations of Algebras (Ottawa, ON, 1992), CarletonOttawa Math. Lecture Note Ser. 14 Carleton Univ., Ottawa, ON, 1992.

[10] H. Derksen and J. Weyman, Semi-invariants of quivers and saturation for LittlewoodRichardson coefficients, J. Amer. Math. Soc. 13 (2000), no. 3, 467-479.

[11] (2002), no. 3, 245-265.

[12] — The combinatorics of quiver representations, Ann. Inst. Fourier (Grenoble) 61 (2011), no. 3, 1061-1131.

[13] N.D. Dichev, Thick subcategories for quiver representations, Ph.D. Thesis, Universität Paderborn, Germany, 2009.

[14] F. Digne, Présentations duales des groupes de tresses de type affine $\widetilde{A}$, Comment. Math. Helv. 81 (2006), no. 1, 23-47.

[15] W. Geigle and H. Lenzing, Perpendicular categories with applications to representations and sheaves, J. Algebra 144 (1991), no. 2, 273-343.

[16] D. Happel, Triangulated categories in the representation theory of finite-dimensional algebras, London Mathematical Society Lecture Note Series 119, Cambridge University Press, Cambridge, 1988.

[17] K. Igusa, K. Orr, G. Todorov, and J. Weyman, Cluster complexes via semi-invariants, Compos. Math. 145 (2009), no. 4, 1001-1034.

[18] K. Igusa and R. Schiffler. Exceptional sequences and clusters, with an appendix by the authors and H. Thomas, J. Algebra 323 (2010), no. 8, 2183-2202. 
[19] C. Ingalls and H. Thomas, Noncrossing partitions and representations of quivers, Compos. Math. 145 (2009), no. 6, 1533-1562.

[20] V.G. Kac, Infinite root systems, representations of graphs and invariant theory, Invent. Math. 56 (1980), 57-92.

[21] _ Infinite root systems, representations of graphs and invariant theory. II., J. Algebra 78 (1982), no. 1, 141-162.

[22] A.D. King, Moduli of representations of finite-dimensional algebras., Quart. J. Math. Oxford Ser. (2) 45 (1994), no. 180, 515-530.

[23] H. Lenzing, Hereditary categories, Handbook of tilting theory, 105-146, London Math. Soc. Lecture Note Ser. 332, Cambridge Univ. Press, Cambridge, 2007.

[24] A. Schofield, Semi-invariants of quivers, J. London Math. Soc. 43 (1991), 383-395.

[25] _ General representations of quivers, Proc. London Math. Soc. (3) 65 (1992), no. 1, 46-64.

[26] A. Schofield and M. Van den Bergh, Semi-invariants of quivers for arbitrary dimension vectors, Indag. Math. (N.S.) 12 (2001), no. 1, 125-138.

[27] D. Simson and A. Skowroński, Elements of the representation theory of associative algebras. Vol. 2., Tubes and concealed algebras of Euclidean type. London Mathematical Society Student Texts 71, Cambridge University Press, Cambridge, 2007.

Colin Ingalls, Dept. of Math. and Stat, University of New Brunswick, Fredericton, NB, CANADA, E3B 5A3

Charles Paquette, Dept. of Math., University of Connecticut, Storrs, CT, USA, 06269-3009

Hugh Thomas, Dept. of Math. and Stat, University of New Brunswick, Fredericton, NB, Canada, E3B 5A3 